\title{
End-of-Life Inventory Decisions for Consumer Electronics Service Parts
}

\author{
M. Pourakbar ${ }^{\dagger}$, J.B.G. Frenk ${ }^{\ddagger}$, R. Dekker ${ }^{\dagger *}$ \\ $†$ Erasmus School of Economics, Erasmus University Rotterdam \\ $\ddagger$ Faculty of Engineering and Natural Sciences, Sabanci University \\ †Burgemeester Oudlaan 50, 3000DR Rotterdam, The Netherlands \\ †Orhanli-Tuzla, 34956 Istanbul, Turkey \\ pourakbar@ese.eur.nl, frenk@sabanciuniv.edu,rdekker@ese.eur.nl \\ * Phone: (+31) 10 - 40 81274, Fax: (+31) 10 - 4089162
}

Econometric Institute Report EI 2009-48

February 24, 2010

\begin{abstract}
We consider a consumer electronics (CE) manufacturer's problem of controlling the inventory of spare parts in the final phase of the service life cycle. The final phase starts when the part production is terminated and continues until the last service contract or warranty period expires. Placing final orders for service parts is considered to be a popular tactic to satisfy demand during this period and to mitigate the effect of part obsolescence at the end of the service life cycle. To satisfy demand for service in the final phase, previous research focuses on repairing defective products by replacing the defective parts with properly functioning spare ones.
\end{abstract}


However, for consumer electronic products there is a remarkable price erosion while repair costs may stay steady over time. As a consequence, this introduces the idea that there might be a point in time at which the unit price of the product is lower than repair associated costs. Therefore, it would be more cost effective to adopt an alternative policy to meet demands for service such as offering customers a replacement of the defective product with a new one or giving a discount on the next generation of the product. This paper examines the cost trade-offs of implementing alternative policies for the repair policy and develops an exact formulation for the expected total cost function. Based on this developed cost function we propose policies to simultaneously find the optimal final order quantity and the time to switch from the repair to an alternative replacement policy. Numerical analysis of a real world case study sheds light over the effectiveness and advantage of these policies in terms of cost reduction and also yields insights into the quantitative importance of the various cost parameters.

Keywords: service parts, end-of-life inventory control, consumer electronics

\section{Introduction}

In this paper, we consider the inventory control of service parts of a consumer electronics (CE) manufacturer in the final phase of their service life cycle. This problem is known in the literature as the end-of-life (EOL) inventory problem. It is also called the final buy problem (FBP), or the end of production problem (EOP). The final phase starts when service part production is terminated and ends when the last service (or warranty) contract expires. In general, the final phase is the longest period within the life cycle of a service part. For instance, in the electronics industry this phase may last from four up to thirty years while the production of electronic appliances is normally terminated after less than two years (Teunter and Klein Haneveld, 2002). In the past decade, there

has been a significant increase in innovation throughout the consumer electronic industry. As a result, a typical product may go through all its life cycle stages including development, initial, normal and final production within a year, or less. Accordingly, this spurt in innovation has led to placement of the final order, that should suffice for the sequel of the service period, typically within 
a year after production kick-off. As a consequence, the difficulty of accurately predicting demand over time and the lack of a sound inventory system to estimate final order quantities have resulted in many cost ineffective purchases. On one hand, companies are mandated to satisfy customer demand for service parts due to warranty obligations, but on the other hand, they face a huge obsolescence and disposal risk at the end of the final phase. Many firms have encountered large write-offs of excess inventory after the product life ultimately ends. For example, IBM disclosed a $\$ 1$ billion loss from its personal-computer business in 1998, attributed to excess PCs in dealer channels that had to be sold at a steep discount (Bulkeley, 1999).

One of the main obstacles in the final phase inventory management is that the acquisition of parts is no longer guaranteed and this forces companies to decide on the final order quantity before the cessation of the production. Basically, during the final phase, customers return defective products for service. The literature relevant to the management of the final phase inventory focuses only on the repair of defective products by replacing the defective part of the product with a functioning one. This functioning part may be either a new part or a repaired returned item. To the best of our knowledge, the literature considers this as the only way to service customers. This approach makes sense as the one and only feasible tool to service the demands for very expensive capital goods and their associated spare parts. However, for consumer electronic goods other approaches should be considered because of the continuous price erosion of such products over time. This price erosion is very typical of consumer electronic goods and may even exceed an annual rate of $30 \%$, while the repair associated costs may stay steady over time. This introduces the idea that within the final phase there may be a break even point in time after which a regular repair policy is no longer the best policy with respect to the total cost. After this break-even point, a firm can satisfy customer demands through an alternative policy such as product swapping or set exchange. Swapping means offering the customer a new product rather than repairing a defective product. From a practical standpoint, there are many cases, particularly for consumer electronic appliances, where swapping is actually more cost-effective than using expensive spare parts to repair defective products. Other alternative policies include offering discount on a new model of the product, or by 
giving credit or monetary compensation to customers.

We contribute to the literature of the end-of-life inventory problem with developing a new methodology introducing the possibility of switching to an alternative policy. According to this methodology, from some point in time to the end of the service life cycle, the system services customers through an alternative policy instead of a repair policy. Throughout this paper we mean by repair policy the whole process of replacement of the defective part of a product with a functioning spare part. We first develop a closed-form expression for the expected total discounted cost function. Then, we propose four policies to deal with this problem. The first is when a manufacturer is loathe to scrapping on-hand serviceable inventory and switches to an alternative policy only when on-hand inventory of service parts is depleted. In this case, the only decision variable is the final order quantity. In the second policy a scrapping option is taken into account. The manufacturer scraps on-hand inventory if it is clear that scrapping the available inventory and switching the policy is more cost efficient. Hence, the decision variables are final order quantity and policy switching time. No inventory level review takes place in these first two approaches. In other words, in the first two approaches the optimal value of the decision variables are calculated just once at the beginning of the period and there is no further adjustment of optimal values after demand realization. To incorporate review, we develop two rolling horizon types of policies. In the third policy, after demand realization given the inventory level at each point in time we recalculate the optimal time to switch. In the fourth policy, given the optimal time to switch we obtain the optimal inventory level at each point in time.

The modeling approach is based on a real world case study. This problem was brought to us by a major European consumer electronic goods manufacturer. The company is a prominent global player in this industry and is one of the companies involved in the European Information and Communication Industry Association (EICTA) in which members have agreed on the length of the service period for different products. In general, the consumer electronics service parts or non-professional service parts comprise the majority of parts stocked in service departments and it is reported that two-thirds of the total stock of spare parts belongs to this category. Although 
our methods are generic, we consider a cathode ray tube (CRT) as the service part to illustrate our approach. CRTs were a relatively expensive and crucial element in TV screens and monitors in the 1990s. Due to the introduction of liquid crystal display (LCD), plasma and organic light emitting diode (OLED) screens, CRTs have become obsolete and to their production has been termiated. For the company, obsolescence of CRTs kept in stock in anticipation of demand for service is an enormous challenge. The demand originates from the large installed base of households that own CRT-based products such as TVs and monitors.

The current approach applied by the company is somewhat complex due to the incorporation of differences between the various regions and agreements with the logistic service providers. The approach basically stems from the ideas mentioned in Teunter and Fortuin (1999) for a nearly cost-optimal final order, where a fixed yearly drop in demand is assumed and a newsvendor type of problem is solved using a predicted repair rate. Neither scrapping before the end of the horizon or an alternative policy are considered. This yields a rather simple problem to be solved. However, consumer electronics market-watch shows that these products may have an annual price erosion of up to $30 \%$. Consequently, the company has an incentive to investigate an alternative policy to service customers in addition to simply purchasing a quantity of stocks to sustain repair activities over a long period of time. In other words, it is keen to examine whether it is feasible to implement an alternative policy and whether this will lead to any cost efficiency.

The remainder of this paper is structured as follows: Section 2 proceeds by providing the relevant literature and position of our paper with respect to the existing literature. Section 3 develops building blocks for a cost-driven model and ends by proposing a closed-form expression in terms of elementary functions for the expected total discounted cost. By applying the results of section 3 , section 4 proposes a variety of end of life policies. In Section 5 a numerical analysis is presented and finally section 6 contains discussion and conclusions. 


\section{Literature Review}

Parts that are used in a product have their own life cycles and might become obsolete before the end of the product life cycle. This lack of synchronization between part and product life cycles has been referred to as "Life cycle mismatch" in the literature (Solomon et al. (2000)). Various strategic decisions can be made on how to deal with this mismatch in order to keep the product in the market. These include substituting another part for the obsolete one, obtaining the obsolete part from an after market manufacturer, redesigning the product, discontinuing the product or purchasing a sufficient volume of the obsolete part to sustain production of the product for its remaining life time; this is called a life-time or a last-time buy (Bradley and Guerrero, (2008)). In this paper, we focus on the last-time or end-of-life buy as a countermeasure tool to cope with the final phase inventory problem.

In general, research on the end-of-life inventory problem in the literature can be divided into three categories: service-driven, cost-driven and forecasting based approaches. In a service-driven approach a service level should be optimized regardless of the cost incurred by the system. However, a cost-driven approach gives a monetary value to the unserved part of the demand by means of back-order or penalty costs, and then adapts a policy to minimize the total cost. Forecasting based approaches ignore production and inventory costs and seek to build a forecasting model to mimic the demand behavior during the final phase. This model is used to predict the total amount of demand in the final phase and this predicted value is used as the final order quantity. In the sequel of this section, we will briefly review the literature and provide examples of these three approaches to the end-of-life inventory problem.

Fortuin $(1980,1981)$ describes a service level approach and addresses non-repairable items or consumable spare parts. He derives a number of curves by which the optimal final order quantity for a given service level can be obtained. He assumes an exponentially decreasing demand pattern and uses a normal approximation to derive expression for several service levels. Another servicedriven approach is developed by van Kooten and Tan (2009) for a system in which parts are subject 
to the risk of condemnation. They build a transient Markovian model to represent the problem for a repairable spare part with a certain repair probability and repair lead time. They also develop some approximate models that allow involvement of further real world characteristics. Using their model, the corresponding optimal final order quantity can be obtained for a given service level.

Basically, a cost-driven approach decides on the quantity purchased by weighing the cost of ordering too many against the cost of buying too few or in other words a news-vendor problem approach. Among this category of works the most pertinent to ours is Teunter and Fortuin (1999). They assume that failed parts can be remanufactured and reused. They find a near optimal solution for the final order quantity and further extend their work by introducing a dispose-down-to level policy. This policy allows unused parts to be removed from stock before the end of the horizon. This approach is applied at Philips Consumer Electronics and results are shown in Teunter and Fortuin (1998). Another cost-driven approach is developed by Teunter and Klein Haneveld (1998). They analyze a multi-part life time problem from an equipment supplier perspective. The equipment supplier permits the machine operator to place a final order for critical parts to keep the machine operational to the end of the horizon. Teunter and Klein Haneveld's model minimizes a firm's internal cost for maintaining an obsolete machine where the failure rate of each part is independent of the failure rates of other parts. In another paper, Teunter and Klein Haneveld (2002) consider the possibility of ordering in the final phase but they assume that if the part is not ordered at the beginning of the final phase, its price will be higher in the later stages. They propose an ordering policy consisting of an initial order-up-to level at the beginning of the final phase followed by a subsequent series of decreasing order-up-to levels for various intervals of the planning horizon.

Cattani and Souza (2003) have developed another cost-driven approach that studies the effect of delaying a last-time buy. They perform a news-vendor analysis which considers overage and underage costs to evaluate the effect of delaying final order placement. Another work in this area is carried by Bradley and Guerrero (2009). They consider different parts of a product becoming obsolete sequentially over product life time wherein a final order for each one has to be placed. 
In the proposed model, it is assumed that once a life-time buy inventory for any part is depleted, manufacturing of the product ceases forever. An exact and two heuristic approaches are developed to solve this problem.

Forecasting based approaches focus on forecasting demand for a discontinued product instead of dealing with the production or inventory problem. To the best of our knowledge, this approach was first developed by Moore (1971). The proposed model forecasts the all-time requirements of consumable service parts in the motor-car industry. By plotting the sales data on a logarithmic scale, the author obtains three families of curves to be common for $85 \%$ of the spare parts considered. Later on, Ritchie and Wilcox (1977) develop a method, using renewal theory, to forecast all-time future demand for spare parts to the moment of the final production run. A more recent work by Hong et. al. (2008) develops a stochastic forecasting model using the number of product sales, the product discard rate, the failure rate of the service part and the replacement probability of the failed part. The model decides on the final order quantity based on the forecasting results.

Our work develops a new cost-driven approach. Several issues distinguish our work in this field. First we develop a model incorporating an alternative service policy and show how this will result in cost efficiency. Secondly, we exploit a more sophisticated and detailed model for the cost function that captures the characteristics of a real world problem more precisely. Furthermore,

according to the case study demand analysis a non-stationary Poisson demand process is shown to better represent the demand behavior and it is used as a basis for our analysis. We also investigate the effect of neglecting the non-stationarity in the demand pattern. Even though these assumptions complicate the problem, we show by applying the standard techniques for martingale theory that it is possible to give elementary expressions for the considered expected total cost function.

\section{Introduction of the Problem and Model}

This section briefly describes the problem whereas the next section provides more details about the cost structure. Throughout this paper, we define the end-of-life inventory problem as the problem 
of finding the final order quantity, $n$, of service parts that covers the demand for a finite service period $[0, T]$ and minimizes the expected total discounted cost function given the possibility of switching to an alternative service policy at time $\tau$ before the end of the horizon. In other words, before time $\tau$ the system operates according to the repair policy and services customers by replacement of the defective part with a spare one. At time $\tau$ the repair policy is terminated and the system switches to an alternative service policy that runs to the end of the horizon. Since this approach to the final phase of service can be characterized by two decision variables, $n$ and $\tau$, we call it a $(n, \tau)$ policy. According to a $(n, \tau)$ policy, a final production batch of size $n$ of spare parts is delivered to the inventory system at the beginning of the final phase. The total provisioning cost is $c_{p} n$ with $c_{p}$ denoting either the purchasing or production cost per part. These spare parts have inventory holding costs $h>0$ per unit per time. For any demand occurring before time $\tau$, with probability $0<q<1$ (independent of the arrival process), the returned defective part can be repaired at repair $\operatorname{cost} c_{r}$. After repairing, this part is placed back into the defective product at a service cost $c_{s}$. If the defective part is not repairable, a new part is taken from the serviceable inventory and is placed into the defective product at service $\operatorname{cost} c_{s}$.

For all non-repairable parts arriving before time $\tau$ for which there are no spare parts available in stock, demand is satisfied through an alternative policy. In this case, the cost to meet demand is $c_{a}+p$ with $p$ a penalty cost that is incurred due to the occurrence of stock-out before the switching time $\tau$. This circumstance is called "forced exchange policy" and $c_{a}$ is the associated alternative policy cost incurred per item. From time $\tau$ to the end of the final phase all demands are serviced through an alternative policy at $\operatorname{cost} c_{a}$. Furthermore, if there is stock available at the switching time $\tau$, all the remaining inventory has to be scrapped at a cost of $c_{s c r}$ per unit. All cost terms and notations are summarized in table 1. In summary, the fundamental assumptions of this model are as follows:

A1: The demand process is assumed to be a non-stationary Poisson process with a decreasing intensity function. In practice it is observed that demand arrival for service parts in the final phase has a declining pattern over time due to the decreasing number of installed bases in the market. 
Table 1: Notations summary

\begin{tabular}{|c|l|}
\hline Notation & Definition \\
\hline$c_{p}$ & Provisioning cost of each part \\
\hline$h$ & Holding cost per item per time \\
\hline$c_{s}$ & Service cost per item \\
\hline$c_{r}$ & Repair cost per repairable item \\
\hline$p$ & Penalty cost per item \\
\hline$c_{a}$ & Alternative policy cost per item \\
\hline$c_{s c r}$ & Scrapping cost per item \\
\hline$q$ & Repair yield factor \\
\hline$\gamma$ & Price erosion factor per time \\
\hline$\delta$ & Discounting factor per time \\
\hline$n$ & Final order quantity \\
\hline$\tau$ & Time to switch to the alternative policy \\
\hline
\end{tabular}

This behavior cannot be characterized by a stationary Poisson process.

A2: The repair lead time is assumed to be negligible. This assumption is common in the remanufacturing literature and makes the analysis tractable (see for example Souza et. al., 2002 and Atasu, Cetinkaya, 2006). Moreover, in the context of this case, the negligibility can be justified because the repair lead times are much shorter than the planning period.

A3: All costs are discounted back to the beginning of the horizon. The exponential unit price erosion factor which applies to the devaluation of alternative policy cost is denoted by $\gamma$ and all other cost terms are exponentially discounted to the beginning of the horizon with discount factor $\delta>0$.

\subsection{Demand Behavior}

While deciding upon the final order quantity and the time to switch to the alternative policy, a highly important aspect is the assumption on demand behavior. The general assumption in our work is that the demand for spare parts is an independent, non-stationary Poisson process. Intuitively, this makes sense, since the number of products available in the market is sufficiently large and diminishes over time and consequently so does the demand for service parts. Sigar (2007) has done a detailed analysis over demand for CE spare parts of a firms including CRT that spans 
an eight year period. In this study, the assumption of a Poisson demand stream was validated and several models regarding the intensity function were introduced and tested. Among the proposed forms, the poly-exponential model given by $\lambda(t)=t^{2} \exp (a-b t)$ with $a$ and $b$ are constant parameters, showed the best performance regarding demand prediction. In this paper we consider the same intensity function. It should be noted that our proposed approach is generic and can be adapted for any type of positive intensity function. With intensity function $\lambda(t)$ the so-called mean

value function is given by $\Lambda(t)=\int_{0}^{t} \lambda(s) d s$. To make the problem analytically tractable, we apply a piecewise linear approximation to the mean value function $\Lambda(t)$ in the analysis. We will elaborate on this issue in the numerical analysis section.

\subsection{Inventory Process}

In this section, we introduce notations and definitions to build up the equations expressing the inventory process. The demand process for spare parts is given by a non-stationary Poisson process defined by $\mathbf{N}_{\Lambda}:=\{\mathbf{N}(\Lambda(t)): t \geq 0\}$ with $\Lambda$ a strictly increasing continuous function and $\mathbf{N}$ a Poisson process with rate 1 . Since $1-q$ is the probability of an arriving defective part being non-repairable, the net stock inventory process of spare parts decreases before the policy switching time, $\tau$, according to a non-stationary Poisson process with a strictly increasing mean value function $\Lambda_{1}(t)=(1-q) \Lambda(t), t \geq 0$. This is due to the well known thinning property of a non-stationary Poisson process (Ross (1970)). Hence it follows that the net inventory process $\mathbf{I N}=\{\mathbf{I N}(t): t \leq \tau\}$ has the form

$$
\mathbf{I N}(t)=n-\mathbf{N}_{\Lambda_{1}}(t) .
$$

Moreover, by relation (1) the hitting time $\sigma_{n}$ at which the net stock process equals zero is given by

$$
\sigma_{n}:=\min \left\{t \geq 0: \mathbf{N}_{\Lambda_{1}}(t) \geq n\right\} .
$$


Since the function $\Lambda$ is strictly increasing and continuous, we obtain that its inverse function $\Lambda^{-1}$ : $[0, \infty) \rightarrow[0, \infty)$ given by

$$
\Lambda^{-1}(s)=\inf \{t \geq 0: \Lambda(t)>s\}
$$

is strictly increasing, continuous and satisfies $\Lambda^{-1}(\Lambda(t))=t$ for every $t \geq 0$. Also, since $\Lambda_{1}(t)=$ $(1-q) \Lambda(t)$ we obtain $\Lambda_{1}^{-1}(s)=\Lambda^{-1}\left((1-q)^{-1} s\right)$. This implies by relation (2) that

$$
\begin{aligned}
\sigma_{n} & =\Lambda_{1}^{-1}\left(\inf \left\{\Lambda_{1}(s) \geq 0: \mathbf{N}\left(\Lambda_{1}(s)\right) \geq n\right\}\right)=\Lambda_{1}^{-1}(\inf \{t \geq 0: \mathbf{N}(t) \geq n\}) \\
& =\Lambda_{1}^{-1}\left(\mathbf{T}_{n}\right)=\Lambda^{-1}\left((1-q)^{-1} \mathbf{T}_{n}\right)
\end{aligned}
$$

with $\mathbf{T}_{n}:=\sum_{k=1}^{n} \mathbf{X}_{k}$ and $\mathbf{X}_{k}$ a sequence of independent and exponentially distributed random variables with parameter 1 . Clearly relation (4) expresses the intuition that the arrival time of the $n$th non-repairable part depends on the inverse of the mean value function of the Poisson arrival process of non-repairable items.

\subsection{Exact Calculation of Expected Discounted Cost Using a Martingale Ap- proach}

In this section, we give an exact calculation of the total expected discounted cost function, $C_{\delta}(n, \tau)$, of a $(n, \tau)$-policy, by showing that a related cost process is a martingale with filtration $\left(\mathcal{F}_{t}\right)_{0 \leq t \leq \infty}$ (see Protter (1992)) and $\tau \wedge \sigma_{n}:=\min \left\{\tau, \sigma_{n}\right\}$ is a stopping time of this process. Using the optional sampling theorem for martingales enables us to simplify our calculations.

To start with the different cost components, let $C_{1, \delta}(n, \tau)$ with discount factor $\delta>0$ be the expected total discounted inventory holding cost of a $(n, \tau)$ policy. The holding cost is discounted back to the beginning of the final phase and the main advantage of this is that capital costs do not have to be included in the holding cost rates. This is especially important in an inventory system with both repair and disposal options. These two options have different associated capital costs. As a result, it is not clear what the right capital holding cost rates should be (see (Teunter et. 
al., 2000) for a detailed discussion of this issue). In our setting holding cost incorporates storage, warehouse material handling, insurance and loss and damage (shrinkage) costs but not capital cost. The system incurs inventory costs either up to time $\tau$ or to the time $\sigma_{n}$ of hitting inventory level 0 (whichever occurs first). Therefore the discounted inventory holding cost is given by

$$
C_{1, \delta}(n, \tau)=h \mathbb{E}\left(\int_{0}^{\tau \wedge \sigma_{n}} \exp (-\delta t) \mathbf{I N}(t) d t\right)
$$

Hence by relation (1) and (5) we obtain

$$
C_{1, \delta}(n, \tau)=h n \delta^{-1}\left(1-\mathbb{E}\left(\exp \left(-\delta\left(\tau \wedge \sigma_{n}\right)\right)\right)\right)-h \mathbb{E}\left(\int_{0}^{\tau \wedge \sigma_{n}} \exp (-\delta t) \mathbf{N}_{\Lambda_{1}}(t) d t\right)
$$

Whenever the system provides service to an end customer by replacing the failed part with a properly functioning one, a service $\cos t c_{s}$ is incurred. This is probably the most difficult cost to estimate among all the costs identified in the process. The problem is usually solved in the literature by ignoring it or claiming that it is negligible compared to the other costs. However, due to high labor costs especially in Europe and North America, it is in fact considerable. Service cost mainly includes labor cost incurred during the diagnosis and replacement of the failed parts. To calculate the expected total discounted service cost, $C_{2, \delta}(n, \tau)$, of a $(n, \tau)$ policy over the planning horizon, we first observe that up to time $\tau \wedge \sigma_{n}$ all arriving defective parts incur a service cost $c_{s}$, while from time $\tau \wedge \sigma_{n}$ up to time $\tau$ this only applies to the repairable parts. Using the thinning property, the arrival process of repairable parts is a non-stationary Poisson process with mean value function $\Lambda_{2}(t)=q \Lambda(t)$ for every $t \geq 0$. This yields

$$
C_{2, \delta}(n, \tau)=c_{s} \mathbb{E}\left(\int_{0}^{\tau \wedge \sigma_{n}} \exp (-\delta t) d \mathbf{N}_{\Lambda}(t)\right)+c_{s} \mathbb{E}\left(\int_{\tau \wedge \sigma_{n}}^{\tau} \exp (-\delta t) d \mathbf{N}_{\Lambda_{2}}(t)\right)
$$

Perceivably in equation (7), the first term represents the service cost for all demands before the inventory depletion time. The second term applies for all repairable parts arriving after the depletion time and before the policy switching time. It is worth noting that the above integral is a 
Riemann-Stieltjes integral (see chapter 1 of Widder, (1972)). Using well-known properties of a non-stationary Poisson process and the definition of a Riemann-Stieltjes integral the expression in relation (7) for the expected total discounted service costs can be rewritten as follows

$$
\begin{aligned}
C_{2, \delta}(n, \tau) & =q c_{s} \int_{0}^{\tau} \exp (-\delta t) d \Lambda(t)+c_{s} \mathbb{E}\left(\int_{0}^{\tau \wedge \sigma_{n}} \exp (-\delta t) d\left(\mathbf{N}_{\Lambda}-\mathbf{N}_{\Lambda_{2}}\right)(t)\right) \\
& =q c_{s} \int_{0}^{\tau} \exp (-\delta t) d \Lambda(t)+c_{s} \mathbb{E}\left(\int_{0}^{\tau \wedge \sigma_{n}} \exp (-\delta t) d \mathbf{N}_{\Lambda_{1}}(t)\right) .
\end{aligned}
$$

Parts repair cost is incurred whenever a repairable defective part is repaired. In most service related research, this cost is assumed to be small compared to other costs and therefore neglected. However, using the same argument as mentioned above with reference to the service cost, it may be worth including in the model. In our setting, repair cost includes labor and freight cost. Freight cost is the cost of transporting the part to the repair shop and then to the service department once repaired. In order to calculate this cost, we first observe using the definition of $\tau$ that the repair policy terminates at time $\tau$. Since with probability $q$ a defective part can be repaired, the arrival process of repairable parts is a non-stationary Poisson process with mean value function $\Lambda_{2}(t)=$ $q \Lambda(t)$ for every $t \geq 0$. Hence the expected total discounted repair cost $C_{3}(n, \tau)$ of a $(n, \tau)$ policy is given by

$$
C_{3, \delta}(n, \tau)=c_{r} \mathbb{E}\left(\int_{0}^{\tau} \exp (-\delta t) d \mathbf{N}_{\Lambda_{2}}(t)\right)=q c_{r} \int_{0}^{\tau} \exp (-\delta t) d \Lambda(t)
$$

From time $\tau, \tau \leq T$, to the end of the final phase period, the EOL process is in the alternative policy state and demands for service are met by an alternative policy instead of the repair policy. But if the inventory level hits zero before $\tau$, the system is forced to switch to the alternative policy earlier for non-repairable parts. This alternative service can be swapping product with a new one, leniency, monetary compensation or an alternative product. Either way, this cost is referred to as the alternative policy costs, $c_{a}$. Alternative service is provided in two circumstances. The first is when there is a stock-out before the policy switching time. In this case, repair policy is still 
less expensive than the alternative policy, implying an (potentially extremely) unpleasant situation. This case of policy switching is referred to as a "forced policy exchange". Additional costs called, "penalty costs" denoted by $p$ are applicable for these exchanges. The reason to include this penalty cost term can be due to customer dissatisfaction or the possibility of a cheaper alternative service only being available at some later time. Moreover, acquiring products before the planned time $\tau$ may impose extra ordering costs to the system. For these reasons, the penalty cost, $p$, is added to the computation and the alternative policy cost per nonrepairable parts arriving before $\tau$ equals $c_{a}+p$. The second circumstance happens when there is demand for service after the policy switching time, $\tau$. In this event called "regular policy exchange", an alternative policy is, in fact, the preferred service policy. For regular policy exchanges, the costs incurred, $c_{a}$, are mostly the cost of running the alternative policy. It should be noted that the costs due to forced exchanges are calculated over the shortages (non-repairable items arrival) before $\tau$, whereas the costs due to policy exchanges are calculated simply over the demand after the policy switching time. Therefore, the total expected discounted penalty and alternative policy costs up to the end of the service period is given by

$$
C_{4, \gamma}(n, \tau)=c_{a} \mathbb{E}\left(\int_{\tau}^{T} \exp (-\gamma t) d \mathbf{N}_{\Lambda}(t)\right)+\left(c_{a}+p\right) \mathbb{E}\left(\int_{\tau \wedge \sigma_{n}}^{\tau} \exp (-\gamma t) d \mathbf{N}_{\Lambda_{1}}(t)\right)
$$

Again applying the well-known properties of a non-stationairy Poisson process we obtain by relation (10) that

$$
C_{4, \gamma}(n, \tau)=\left\{\begin{array}{l}
c_{a} \int_{\tau}^{T} \exp (-\gamma t) d \Lambda(t)+(1-q)\left(c_{a}+p\right) \int_{0}^{\tau} \exp (-\gamma t) d \Lambda(t) \\
-\left(c_{a}+p\right) \mathbb{E}\left(\int_{0}^{\tau \wedge \sigma_{n}} \exp (-\gamma t) d \mathbf{N}_{\Lambda_{1}}(t)\right)
\end{array}\right.
$$

When all service obligations have ended or when the switching time has arrived, the stock on hand turns into excess stock and has to be removed from the warehouse. Scrapping costs include disposal, transportation and environmental cost since most countries heavily tax disposal of stock. It is worth noting that in some cases, excess stock can be sold to a third party at a salvage value. In such a situation, the corresponding scrapping cost is negative since it generates revenue. The 
system only incurs scrapping cost in case $\tau \leq \sigma_{n}$, which means there are serviceable items available in inventory at the policy switching time. Using using relation (1) together with the fact that $\mathbf{I N}\left(\tau \wedge \sigma_{n}\right)=0$ for $\tau \geq \sigma_{n}$, then we have

$$
\begin{aligned}
C_{5, \delta}(n, \tau) & =c_{s c r} \mathbb{E}\left(\exp \left(-\delta\left(\tau \wedge \sigma_{n}\right)\right) \mathbf{I N}\left(\tau \wedge \sigma_{n}\right)\right) \\
& =n c_{s c r} \mathbb{E}\left(\exp \left(-\delta\left(\tau \wedge \sigma_{n}\right)\right)\right)-c_{s c r} \mathbb{E}\left(\exp \left(-\delta\left(\tau \wedge \sigma_{n}\right)\right) \mathbf{N}_{\Lambda_{1}}\left(\tau \wedge \sigma_{n}\right)\right)
\end{aligned}
$$

The last cost term that needs to be calculated for a $(n, \tau)$ policy is the final order provisioning cost. This is either purchasing or production cost incurred for $n$ parts and is calculated by:

$$
C_{6, \delta}(n, \tau)=c_{p} n
$$

Adding the individual cost components listed in relations (6), (8), (11) (9), (12) and (13) we finally obtain the average discounted $\operatorname{cost} C_{\delta, \gamma}(n, \tau)$ of a $(n, \tau)$ policy given by

$$
C_{\delta, \gamma}(n, \tau)=\sum_{i=1, i \neq 4}^{6} C_{i, \delta}(n, \tau)+C_{4, \gamma}(n, \tau)
$$

Looking in more detail to these individual cost components we still need to simplify four different expectations. The following lemma shows that this task can be reduced to three different expectations. In the rest of this paper only the results are mentioned and all the proofs are listed in the Appendix.

\section{Lemma 1 It follows}

$$
\begin{aligned}
& \delta \mathbb{E}\left(\int_{0}^{\tau \wedge \sigma_{n}} \exp (-\delta t) \mathbf{N}_{\Lambda_{1}}(t) d t\right) \\
= & \mathbb{E}\left(\int_{0}^{\tau \wedge \sigma_{n}} \exp (-\delta v) d \mathbf{N}_{\Lambda_{1}}(v)\right)-\mathbb{E}\left(\exp \left(-\delta\left(\tau \wedge \sigma_{n}\right) \mathbf{N}_{\Lambda_{1}}\left(\tau \wedge \sigma_{n}\right)\right) .\right.
\end{aligned}
$$


Proof. See appendix.

To simplify the notational burden, for $\alpha \geq 0$ we introduce the expectations

$$
\begin{gathered}
J_{1}(\alpha, n, \tau):=\mathbb{E}\left(\exp \left(-\alpha\left(\tau \wedge \sigma_{n}\right)\right)\right) \\
J_{2}(\alpha, n, \tau):=\mathbb{E}\left(\int_{0}^{\tau \wedge \sigma_{n}} \exp (-\alpha t) d \mathbf{N}_{\Lambda_{1}}(t)\right)
\end{gathered}
$$

and

$$
J_{3}(\alpha, n, \tau):=\mathbb{E}\left(\exp \left(-\alpha\left(\tau \wedge \sigma_{n}\right)\right) \mathbf{N}_{\Lambda_{1}}\left(\tau \wedge \sigma_{n}\right)\right)
$$

Applying Lemma 1 and relation (14) we obtain after rearranging the expressions for the different cost components in relations (6), (8), (11) (9), (12) and (13) that

$$
C_{\delta, \gamma}(n, \tau)=K(n, \tau)+\sum_{i=1}^{3} \kappa_{i} J_{i}(\delta, n, \tau)+\kappa_{4} J_{2}(\gamma, n, \delta)
$$

with

$$
K(n, \tau)=\left\{\begin{array}{l}
q\left(c_{s}+c_{r}\right) \int_{0}^{\tau} \exp (-\delta t) d \Lambda(t)+(1-q)\left(c_{a}+p\right) \int_{0}^{\tau} \exp (-\gamma t) d \Lambda(t) \\
+c_{a} \int_{\tau}^{T} \exp (-\gamma t) d \Lambda(t)+n\left(h \delta^{-1}+c_{p}\right)
\end{array}\right.
$$

and $\kappa_{1}:=n\left(c_{s c r}-h \delta^{-1}\right), \kappa_{2}:=c_{s}-h \delta^{-1}, \kappa_{3}:=h \delta^{-1}-c_{s c r}$ and $\kappa_{4}:=-\left(c_{a}+p\right)$.

Considering relation (19) we still need to evaluate the three different expectations listed in (16) up to (18). To start with the first expectation we observe that

$$
J_{1}(\alpha, n, \tau)=\exp (-\alpha \tau) \mathbb{P}\left\{\sigma_{n}>\tau\right\}+\mathbb{E}\left(\exp \left(-\alpha \sigma_{n}\right) \mathbf{1}_{\left\{\sigma_{n} \leq \tau\right\}}\right)
$$

with $\mathbf{1}_{A}$ denting the Bernoulli indicator random variable of the event $A$. This shows by relation (4) that

$$
J_{1}(\alpha, n, \tau)=\exp (-\alpha \tau) \mathbb{P}\left\{\mathbf{T}_{n}>\Lambda_{1}(\tau)\right\}+\mathbb{E}\left(\exp \left(-\alpha \Lambda_{1}^{-1}\left(\mathbf{T}_{n}\right)\right) \mathbf{1}_{\left\{\mathbf{T}_{n} \leq \Lambda_{1}(\tau)\right\}}\right)
$$


To simplify the expectation $J_{2}(\alpha, n, \tau)$ we next show that a related process is a martingale.

Lemma 2 If $\mathbf{N}_{\Phi}$ is a (non-stationary) Poisson process with continuous mean value function $\Phi$, then for every $\alpha \geq 0$ the stochastic process $\mathbf{M}=\left\{\mathbf{M}_{t}: t \geq 0\right\}$ given by

$$
\mathbf{M}_{t}=\int_{0}^{t} \exp (-\alpha v) d \mathbf{N}_{\Phi}(v)-\int_{0}^{t} \exp (-\alpha v) d \Phi(v)
$$

is a martingale with respect to filtration $\left(\mathcal{F}_{t}\right)_{0 \leq t \leq \infty}$.

Proof. See appendix.

Applying Lemma 2 and using Doob's optional sampling theorem for martingales (See Theorem 18 of Protter, (1992)) the next lemma yields a simpler expression for the expectation $J_{2}(\alpha, n, \tau)$.

Lemma 3 It follows that

$$
J_{2}(\alpha, n, \tau)=(1-q) \mathbb{E}\left(\int_{0}^{\tau \wedge \sigma_{n}} \exp (-\alpha t) d \mathbf{N}_{\Lambda}(t)\right)
$$

and

$$
\mathbb{E}\left(\int_{0}^{\tau \wedge \sigma_{n}} \exp (-\alpha t) d \mathbf{N}_{\Lambda}(t)\right)=\mathbb{E}\left(\int_{0}^{\Lambda(\tau) \wedge(1-q)^{-1} \mathbf{T}_{n}} \exp \left(-\alpha \Lambda^{-1}(t)\right) d t\right) .
$$

Proof. See appendix.

Finally we still need to evaluate $J_{3}(\alpha, n, \tau)$.

\section{Lemma 4 It follows}

$$
J_{3}(\alpha, n, \tau)=\exp (-\alpha \tau) \mathbb{E}\left(\mathbf{N}_{\Lambda_{1}}(\tau) \mathbf{1}_{\left\{\mathbf{N}_{\Lambda_{1}}(\tau) \leq n-1\right\}}\right)+n \mathbb{E}\left(\exp \left(-\alpha \Lambda_{1}^{-1}\left(\mathbf{T}_{n}\right)\right) \mathbf{1}_{\left\{\mathbf{T}_{n} \leq \Lambda_{1}(\tau)\right\}}\right)
$$

Proof. See appendix.

Applying now relations (19), (20) and Lemma 3 and 4 the total discounted cost of a $(n, \tau)$ 
policy is given by

$C_{\delta, \gamma}(n, \tau)=\left\{\begin{array}{l}\beta_{1} \mathbb{E}\left(\int_{0}^{\Lambda(\tau) \wedge(1-q) \mathbf{T}_{n}} \exp \left(-\delta \Lambda^{-1}(t) d t\right)+\beta_{2} \mathbb{E}\left(\int_{0}^{\Lambda(\tau) \wedge(1-q) \mathbf{T}_{n}} \exp \left(-\gamma \Lambda^{-1}(t) d t\right)\right.\right. \\ +\beta_{3} \mathbb{P}\left\{\mathbf{T}_{n}>(1-q) \Lambda(\tau)\right\}+\beta_{4} \mathbb{E}\left(\mathbf{N}_{\Lambda_{1}}(\tau) \mathbf{1}_{\left\{\mathbf{N}_{\Lambda_{1}}(\tau) \leq n-1\right\}}\right)+K(n, \tau)\end{array}\right.$

with $\beta_{1}=\left(c_{s}-h \delta^{-1}\right)(1-q), \beta_{2}=-\left(c_{a}+p\right)(1-q), \beta_{3}=n\left(c_{s c r}-h \delta^{-1}\right) \exp (-\delta \tau)$ and $\beta_{4}=\left(h \delta^{-1}-c_{s c r}\right) \exp (-\delta \tau)$.

Hence we have reduced the computation of the discounted cost to elementary integrals, which can be calculated.

\section{End-of-Life Inventory Policies}

Using the results of the previous section, we now propose a variety of end-of-life policies based on various settings. Firstly, these settings consider the possibility of implementing a scrapping operation. In general, the company is loathe to scrap parts. Therefore, in our first policy, this unwillingness to scrap is taken into account and it is assumed that an alternative policy is triggered once the serviceable inventory depletes. In this case, the only decision variable is the final order quantity, $n$. In the second policy, the serviceable inventory can be scrapped at the switching time, $\tau$. Accordingly, the decision variables are $n$ and $\tau$. Contrary to the company's intuition, the numerical analysis shows that this policy leads to a better cost efficiency compared to the one without scrapping. The second setting is related to the inclusion of a review process. The first two policies are intrinsically static decision making processes meaning that decision making is performed once at the beginning of the horizon and values of the decision variables are set based on the outcome of the optimization procedure. But during the course of the final phase, demand may fluctuate more or less than expected. Therefore, we add a review process to the $(n, \tau)$ policy (the second one). In this review process, at the beginning of each period we re-visit the available serviceable inventory on-hand and based on that we re-optimize the policy switching time (as 
implemented in the third policy). In the fourth policy, we keep the policy switching time fixed, as obtained according to the second policy, and find the optimal inventory level at the beginning of each period. Furthermore, we consider the option that the system can partially scrap the excessive inventory if it exceeds the optimal inventory level. The latter two policies are inherently rollinghorizon type of policies which aim at improving the second one.

It is worth mentioning that in these policies the time-line is divided into equi-spaced intervals to which we limit our attention. Then, for the second and third policies, we consider each interval to be continuous in time and use the lemmas presented in the previous section in each interval to calculate the corresponding cost terms.

\subsection{One-time Buy, without Review or Scrapping}

If the company is not willing to dispose of any serviceable inventory, a "one-time buy without review or scrapping" policy can be applied. The final order is placed at the beginning of the period and the alternative policy is triggered once the inventory depletes. Hence, no scrapping cost occurs before $T$, and it may happen only at the end of the horizon if there is any stock left. This policy resembles the Teunter and Fortuin (1998) end-of-life inventory problem since the only decision variable is the final order quantity. We can apply a marginal cost analysis in order to find the optimal $n$. We define $\mathbb{E} \Delta T C(n)=\mathbb{E}(T C(n))-\mathbb{E}(T C(n-1))$ as the cost difference of ordering $n-1$ instead of $n$. Then, we can find $\mathbb{E} \Delta T C(n)$ by conditioning on $\sigma_{n}$. If the inventory level drops to zero before the end of the horizon , $\sigma_{n} \leq T$, the overage cost of carrying $n$ items instead of $n-1$ includes purchasing, holding, service and repair cost but the system may benefit from less alternative policy cost. Hence we have the following

$$
\mathbb{E} \Delta T C\left(n \mid \sigma_{n} \leq T\right)=c_{p}+h \int_{0}^{\sigma_{n}} \exp (-\delta t) d t-c_{a} \exp \left(-\gamma \sigma_{n}\right)+\left(q c_{r}+c_{s}\right) \exp \left(-\delta \sigma_{n}\right)
$$

Next, we consider a case that $\sigma_{n}>T$. This happens when the system keeps serviceable inventory available for the entire final phase. In order to calculate $\mathbb{E} \Delta T C(n)$ in this case, we have to include 
extra purchasing cost for one unit, holding cost for period $(0, T)$ and extra scrapping cost at time $T$. Therefore we have

$$
\mathbb{E} \Delta T C\left(n \mid \sigma_{n}>T\right)=c_{p}+h \int_{0}^{T} \exp (-\delta t) d t+c_{s c r} \exp (-\delta T)
$$

Using relations (26) and (27) we have

$$
\begin{aligned}
\mathbb{E} \Delta T C(n) & =\mathbb{E} \Delta T C\left(n, \sigma_{n} \leq T\right)+\mathbb{E} \Delta T C\left(n, \sigma_{n}>T\right) \\
& =c_{p}+\frac{h}{\delta}+\left(q c_{r}+c_{s}-\frac{h}{\delta}\right) \int_{0}^{T} \mathbb{P}\left\{\sigma_{n}=t\right\} \exp (-\delta t) d t \\
& -c_{a} \int_{0}^{T} \mathbb{P}\left\{\sigma_{n}=t\right\} \exp (-\gamma t) d t-\left(\frac{h}{\delta}-c_{s c r}\right) \mathbb{P}\left\{\sigma_{n}>T\right\} \exp (-\delta T) .
\end{aligned}
$$

Considering time to be continuous, we can find $n$ as the integer number that satisfies $\mathbb{E} \Delta T C(n) \leq$ 0 and $\mathbb{E} \Delta T C(n+1) \geq 0$.

\subsection{One-time Buy, with Scrapping but without Review}

In this policy, we assume that the system is allowed to scrap items at policy switching time. In other words, if the system carries stock at the time to switch to the alternative policy, all available inventory should be scrapped at that time. We aim at finding the optimal final order quantity, $n$, and the time to switch to the alternative policy, $\tau$.

\subsubsection{Dynamic Programming Approach}

In this section, we develop a backward dynamic program to find the optimal arguments of a $(n, \tau)$ policy. We first divide the time-line into equi-spaced intervals such as $[0,1), \ldots,[T-2, T-1),[T-$ $1, T]$. The starts of intervals and stock on hand at time $t$ are considered as stages and system state respectively. Assume that the stock on hand at time $l$ is $y, y=0,1,2, \ldots$ Let $C_{l}(y, \tau)$ be the total expected cost, corresponding to a $(y, \tau)$ policy in interval $[l, T]$, discounted back to time $l$.

Obviously, considering a backward dynamic program, the cost function $C_{l}(y, \tau)$ is dependent on 
the decision made at time $l+1$. Meaning that if the system keeps on repairing at time $l+1$, it is only allowed to repair in period $l$ since it cannot later switch from the alternative policy to the repair policy. Moreover, if the system decides to switch to the alternative policy at time $l+1$, it can either execute the repair policy during the $l^{\text {th }}$ period and switch to the alternative policy at time $l+1$ or start the alternative policy at time $l$. Therefore, in order to derive the iterative function for each stage, the system needs to decide whether to launch the alternative policy or keep on servicing customers by the repair policy and hence postpone the decision of launching the alternative policy to the next period. If the system switches to the alternative policy, the available stock must be scrapped. Therefore, the expected alternative policy cost from time $l$ up to time $T$ is given by

$$
C_{A L T}(y, l)=c_{s c r} \mathbb{E}(\exp (-\delta l) \mathbf{I N}(l))+c_{a} \mathbb{E}\left(\int_{l}^{T} \exp (-\gamma t) d \mathbf{N}_{\Lambda}(t)\right)
$$

If the system keeps on repairing and the decision to switch to the alternative policy is deferred to the next period, we have relation (30) as the expected repair policy cost for the period $[l, l+1]$. It is the sum of expected holding, service, repair and forced alternative policy exchange costs.

$$
\begin{aligned}
C_{R}(y, l)= & h \mathbb{E}\left(\int_{l}^{l_{f}} \exp (-\delta t) \mathbf{I N}(t) d t\right)+c_{s} \mathbb{E}\left(\int_{l}^{l_{f}} \exp (-\delta t) d \mathbf{N}_{\Lambda}(t)\right) \\
& +c_{s} \mathbb{E}\left(\int_{l_{f}}^{l+1} \exp (-\delta t) d \mathbf{N}_{\Lambda_{2}}(t)\right)+c_{r} \mathbb{E}\left(\int_{l}^{l+1} \exp (-\delta t) d \mathbf{N}_{\Lambda_{2}}(t)\right) \\
& +\left(c_{a}+p\right) \mathbb{E}\left(\int_{l_{f}}^{l+1} \exp (-\gamma t) d \mathbf{N}_{\Lambda_{1}}(t)\right) .
\end{aligned}
$$

In this formula $l_{f}$ is the minimum time wherein either the system runs out of stock or enters the next stage and it is defined as

$$
l_{f}=(l+1) \wedge \min \left\{t \geq l: \mathbf{N}_{\Lambda_{1}}(t) \geq n\right\} .
$$


Hence, the iterative objective function can be defined as

$$
C_{l}(y, \tau)=\min \left\{C_{A L T}(y, l), C_{R}(y, l)+C_{l+1}(y /, \tau)\right\}: y=0,1,2, \ldots
$$

where $y /$ is the expected stock on hand at the beginning of the next period. It is worth noting that the provisioning cost should also be incorporated in the expected total cost function at the beginning of the final phase. Therefore, the repair policy cost at the beginning of the final phase, time 0 , needs to be reformulated as follows

$$
\begin{aligned}
C_{R}(y, 0)= & h \mathbb{E}\left(\int_{0}^{l_{f}} \exp (-\delta t) \mathbf{I N}(t) d t\right)+c_{s} \mathbb{E}\left(\int_{0}^{l_{f}} \exp (-\delta t) d \mathbf{N}_{\Lambda}(t)\right) \\
& +c_{s} \mathbb{E}\left(\int_{l_{f}}^{1} \exp (-\delta t) d \mathbf{N}_{\Lambda_{2}}(t)\right)+c_{r} \mathbb{E}\left(\int_{0}^{1} \exp (-\delta t) d \mathbf{N}_{\Lambda_{2}}(t)\right) \\
& +\left(c_{a}+p\right) \mathbb{E}\left(\int_{l_{f}}^{1} \exp (-\gamma t) d \mathbf{N}_{\Lambda_{1}}(t)\right)+c_{p} y
\end{aligned}
$$

Where $l_{f}=1 \wedge \min \left\{t \geq 0: \mathbf{N}_{\Lambda_{1}}(t) \geq n\right\}$. Relations (29), (30) and (33) can be simplified using lemmas 1-4. The idea is that the time-line is divided into equi-spaced intervals and we consider the beginning of each interval as the potential switching time to the alternative policy. Then each interval is analyzed in a continuous time way by applying the results obtained in section 4 .

So far, we have proposed two policies to deal with the end-of-life inventory problem that can switch to an alternative policy. However, the drawback of these approaches is that there is no further review to revise decision variables based on the state of the system after demand realization. In the next two subsections, two more policies are proposed taking review into account.

\subsection{One-time Buy, with Review and Scrapping}

In this case, the system starts with the optimal arguments for a $(n, \tau)$ policy, gained by the second approach. This policy is a rolling-horizon one in which optimal switching time is recalculated after demand realization in each period. According to this policy, we can determine all possible switching times and find the optimal solution in each period, for a given inventory level $y$ at time 
$t \leq T$. This can be implemented because the expected total cost function of a $(n, \tau)$ policy can be calculated using Lemmas 1-4. If the system is at the beginning of period $t$ and the serviceable inventory on-hand is $y$ then we can calculate the expected total discounted cost for all possible values of $\tau, \tau=t, t+1, \ldots, T$ by the following

$$
\begin{aligned}
C_{\delta, \gamma}(y, l) & =\sum_{l=t}^{\tau}\left(\left(h \mathbb{E}\left(\int_{l}^{l_{f}} \exp (-\delta t) \mathbf{I N}(t) d t\right)+c_{s} \mathbb{E}\left(\int_{l}^{l_{f}} \exp (-\delta t) d \mathbf{N}_{\Lambda}(t)\right)\right.\right. \\
& \left.\left.+c_{r} \mathbb{E}\left(\int_{l}^{l_{f}} \exp (-\delta t) d \mathbf{N}_{\Lambda_{2}}(t)\right)+\left(c_{a}+p\right) \mathbb{E}\left(\int_{l_{f}}^{\tau} \exp (-\gamma t) d \mathbf{N}_{\Lambda_{1}}(t)\right)\right)\right) \\
& +c_{s c r} \mathbb{E}(\exp (-\delta \tau) \mathbf{I N}(\tau))+c_{a} \mathbb{E}\left(\int_{\tau}^{T} \exp (-\gamma t) d \mathbf{N}_{\Lambda}(t)\right)
\end{aligned}
$$

Then, the $\tau$ that leads to the least total cost is selected as the new policy switching time. In this case $l_{f}$ is defined as the minimum time that the system faces a stock-out, enters the next stage or reaches the policy switching time. It is defined by $l_{f}=\min \left\{t \geq l: \mathbf{N}_{\Lambda_{1}}(t) \geq n\right\} \wedge(l+1) \wedge \tau$.

\subsection{One-time Buy, with Review and Partial Scrapping}

As mentioned earlier, in the second policy, decisions over the values of $n$ and $\tau$ are just made once at the beginning of the horizon. During the course of the final phase, the system may encounter less demand than expected, which means that excess stock has to be carried over time. To avoid the build-up of excessive net stock, if the inventory level at time $t_{0}$ is $\mathbf{I N}\left(t_{0}\right)$, the system is given the option to scrap down to level $s, s<\mathbf{I N}\left(t_{0}\right)$. This is called "partial scrapping" since the system can scrap a portion of the serviceable inventory. In this policy, the system starts with the optimal solution, $n$ and $\tau$, obtained by the second policy and then the optimal inventory level is derived by a marginal cost analysis approach. It is a type of news-vendor formulation wherein the marginal profit of scrapping down to level $s$ should compensate the cost of its implementation. If some parts are scrapped, the system benefits from less holding and service costs for the rest of the period but might incur more scrapping and forced policy exchange cost.

Defining $\sigma_{s}$ as the time that inventory level hits zero, we drive the news-vendor equation by 
conditioning on $\sigma_{s}$. $\mathbb{E} \Delta(T C(s))=\mathbb{E}(T C(s))-\mathbb{E}(T C(s-1))$ is defined as the cost difference of scrapping to $s-1$ instead of $s$. In a situation that inventory level drops to zero before $\tau, \sigma_{s} \leq \tau$, the system benefits from less scrapping and forced policy exchange costs but incurs more repair, service and holding costs as shown in the following relation

$$
\begin{aligned}
\mathbb{E}\left(\Delta T C(s) \mid \sigma_{s} \leq \tau\right)= & h \mathbb{E}\left(\int_{t_{0}}^{\sigma_{s}} \exp (-\delta t) d t\right)+\left(q c_{r}+c_{s}\right) \exp \left(-\delta \sigma_{s}\right) \\
& -\left(c_{a}+p\right) \exp \left(-\gamma \sigma_{s}\right)-c_{s c r} \exp \left(-\delta t_{0}\right) .
\end{aligned}
$$

If $\sigma_{s}>\tau$, the system incurs more holding cost but benefits from less scrapping cost at the policy switching time, hence

$$
\mathbb{E}\left(\Delta T C(s) \mid \sigma_{s}>\tau\right)=h \mathbb{E}\left(\int_{t_{0}}^{\tau} \exp (-\delta t) d t\right)-c_{s c r}\left(\exp \left(-\delta t_{0}\right)-\exp (-\delta \tau)\right)
$$

Therefore $s$ is the value satisfying $\mathbb{E} \Delta T C(s+1) \geq 0$ and $\mathbb{E} \Delta T C(s) \leq 0$ where $\mathbb{E} \Delta T C(s)$ is given by

$$
\begin{aligned}
\mathbb{E} \Delta(T C(s))= & \mathbb{E} \Delta\left(T C(s) \mid \sigma_{s} \leq \tau\right) \mathbb{P}\left\{\sigma_{s} \leq \tau\right\}+\mathbb{E} \Delta\left(T C(s) \mid \sigma_{s}>\tau\right) \mathbb{P}\left\{\sigma_{s}>\tau\right\} \\
= & \exp \left(-\delta t_{0}\right)\left(\frac{h}{\delta}-c_{s c r}\right)+\left(q c_{r}+c_{s}-\frac{h}{\delta}\right) \int_{t_{0}}^{\tau} \mathbb{P}\left\{\sigma_{s}=t\right\} \exp (-\delta t) d t \\
& -\left(c_{a}+p\right) \int_{t_{0}}^{\tau} \mathbb{P}\left\{\sigma_{s}=t\right\} \exp (-\gamma t) d t-\left(\frac{h}{\delta}-c_{s c r}\right) \mathbb{P}\left\{\sigma_{s}>\tau\right\} \exp (-\delta \tau) .
\end{aligned}
$$

\section{Numerical Experiments}

We hope to achieve three goals with our numerical experiments. First, we examine which of the proposed policies outperforms others, next we investigate how different cost terms affect the optimal solutions and which of these play a prominent role. Thirdly, we investigate the cost efficiency that can be achieved by an accurate demand forecasting. The data originate from a case study on a 
typical CE service part, namely Cathode Ray Tube, which was an important and expensive part in the former generation of TVs and monitors. The cost structure of the base case scenario is shown in table 2. It is worth noting that in this case swapping is considered as the alternative policy. Accordingly, after the policy switching time customers are offered a new product rather the repairing the defective product.

\subsection{Effects of Cost Parameters}

We first proceed by evaluating the performance of the proposed policies and analyze the sensitivity of the solutions to the different variations of the base case scenario parameters. The planning horizon covers more than five years. These are divided into 66 periods, each of which is assumed to represent one month. Costs are all discounted to the beginning of the final phase. According to the demand analysis (Sigar, 2007), the parameters $a$ and $b$ in the poly-exponential intensity function, given by $\lambda(t)=t^{2} \exp (a-b t)$, have the value 0.2 and 2 respectively. These parameters match the data of the demand for CRT over an eight-year period. The mean value function, $\Lambda(s)=\int_{0}^{s} \lambda(t) d t$, is linearized in each period (month). To this end, after dividing the time-line into equi-spaced intervals, the upper and lower points in each interval are extracted and the mean value function is approximated by the line crossing those two points. This linearization allows us to analytically calculate the cost terms.

The third and fourth policies are rolling-horizon type of approaches, we implemented a simulation program replicating 100 times. The choice of 100 is made on the basis of maintaining a small standard deviation for the expected total cost that reduces the length of the confidence interval to 1000 , in a $90 \%$ confidence interval. Moreover, in order to generate arrival times that follow a non-stationary Poisson process, we use the approach proposed by Cinlar (1975, pp. 94-101). This approach can be implemented due to the linear approximation of $\Lambda(t)$ that makes the $\Lambda^{-1}(t)$ easy to calculate. Average values are shown in Table 2. We investigate the effect of discounting, price erosion and repair yield factors as well as purchasing, holding, alternative policy, repair, scrapping and service costs. 
Table 2: Parameter setting of the base case scenario (in euro)

\begin{tabular}{|l|c|}
\hline Term & Cost \\
\hline Provisioning & 225 \\
\hline Holding & 3.25 \\
\hline Service & 30 \\
\hline Repair & 20 \\
\hline Penalty & 20 \\
\hline Alternative Policy & 645 \\
\hline Scrapping & 30 \\
\hline Price erosion factor per month & 0.02 \\
\hline Discounting factor per month & 0.005 \\
\hline Part repair yield & 0.1 \\
\hline
\end{tabular}

It is observed that the first policy, one time buy without review and without scrapping, shows the worst performance. Therefore, it is considered as the benchmark policy to compare the performance of all policies. The percentage of relative cost improvement that can be achieved by implementing each of the policies compared to the benchmark policy is shown in the $\Delta \%$ column. It can be observed that the policy with partial scrapping outperforms all other policies. The primary factor that leads to the cost efficiencies in this case is that in case of excess stock in each period, the system avoids additional holding and service costs by partially scrapping serviceable inventory down to the optimal level.

The effect of discounting and price erosion factors on decision variables is illustrated in figure 1. Figures 1.a and 1.b show that when the discounting factor increases the time to switch as well as the final order quantity increase. The intuition behind these graphs is that a high discount rate makes the future repair related costs cheaper and thus the system tends to run the repair policy for a longer period. Therefore, the associated final order quantity increases and the time to switch to the alternative policy is postponed to a later time. Increasing $\delta$ from 0.005 to 0.03 results in the increase of final order quantity from 93 to 107 and consequently switching time changes from 22 to 41. Figure 1.c and 1.d show the effect of increasing price erosion on the final order quantity and the time to switch to the alternative policy. As intuition dictates, figure 1.b illustrates when price erosion rate increases the system shows a tendency to start the alternative policy earlier and 
Table 3: Sensitivity analysis

\begin{tabular}{|c|c|c|c|c|c|c|c|c|c|}
\hline \multicolumn{2}{|c|}{} & \multicolumn{2}{c|}{ 1st Policy } & \multicolumn{2}{|c|}{ 2nd Policy } & \multicolumn{2}{c|}{ 3rd Policy } & 4th Policy \\
\hline Parameter & Value & $n$ & cost & $n$ & $\tau$ & $(\Delta) \%$ & $\tau$ & $(\Delta) \%$ & $(\Delta) \%$ \\
\hline$\delta$ & 0.001 & 79 & 31408 & 92 & 21 & 6.9 & 23 & 7.5 & 8.2 \\
& 0.005 & 83 & 30460 & 93 & 22 & 4.4 & 28 & 5.8 & 6.7 \\
& 0.015 & 85 & 29889 & 97 & 24 & 3.5 & 33 & 4.9 & 5.3 \\
& 0.025 & 86 & 29444 & 102 & 34 & 3.2 & 35 & 4.4 & 4.7 \\
\hline$\gamma$ & 0.01 & 85 & 31317 & 97 & 23 & 5.2 & 29 & 5.5 & 6.9 \\
& 0.05 & 79 & 27856 & 87 & 19 & 1.5 & 23 & 4.1 & 5.5 \\
& 0.09 & 72 & 25346 & 75 & 16 & 1.0 & 19 & 3.2 & 4.3 \\
& 0.13 & 61 & 22881 & 64 & 15 & 0.4 & 14 & 2.7 & 3.7 \\
\hline$q$ & 0.0 & 101 & 31911 & 101 & 21 & 1.4 & 23 & 3.0 & 4.2 \\
& 0.2 & 71 & 28204 & 85 & 23 & 4.3 & 27 & 6.4 & 6.9 \\
& 0.4 & 50 & 22719 & 64 & 26 & 4.2 & 30 & 5.5 & 7.1 \\
& 0.8 & 24 & 11153 & 39 & 32 & 5.5 & 27 & 7.2 & 8.6 \\
\hline$c_{p}$ & 100 & 87 & 18130 & 101 & 26 & 6.0 & 27 & 6.3 & 7.8 \\
& 350 & 78 & 42210 & 89 & 22 & 4.1 & 24 & 5.8 & 6.7 \\
& 500 & 67 & 53736 & 72 & 16 & 2.0 & 19 & 4.1 & 5.1 \\
\hline$c_{a}$ & 345 & 71 & 27185 & 79 & 19 & 3.2 & 25 & 4.5 & 7.1 \\
& 945 & 87 & 32464 & 99 & 24 & 7.1 & 27 & 8.5 & 9.1 \\
& 1245 & 90 & 33647 & 101 & 25 & 7.3 & 30 & 9.3 & 9.5 \\
\hline$c_{s}$ & 0 & 84 & 27725 & 96 & 22 & 5.8 & 33 & 6.4 & 7.8 \\
& 10 & 83 & 28636 & 95 & 22 & 5.3 & 32 & 7.5 & 7.6 \\
& 50 & 83 & 32283 & 94 & 22 & 3.6 & 33 & 4.2 & 6.4 \\
\hline$h$ & 0.25 & 88 & 28340 & 98 & 41 & 3.3 & 63 & 4.1 & 5.7 \\
& 6.25 & 80 & 32072 & 92 & 18 & 4.4 & 27 & 5.8 & 6.6 \\
& 9.25 & 78 & 33388 & 90 & 16 & 4.8 & 23 & 6.2 & 7.8 \\
\hline$c_{r}$ & 0 & 83 & 30247 & 95 & 22 & 4.4 & 26 & 5.4 & 6.2 \\
& 5 & 83 & 30300 & 95 & 22 & 4.4 & 27 & 5.1 & 5.4 \\
& 35 & 83 & 30619 & 95 & 22 & 4.4 & 27 & 4.8 & 6.8 \\
\hline$c_{s c r}$ & -30 & 84 & 30451 & 96 & 22 & 4.7 & 25 & 5.2 & 6.7 \\
& 10 & 83 & 30457 & 95 & 22 & 4.5 & 25 & 5.1 & 7.1 \\
& 50 & 83 & 30462 & 94 & 22 & 4.3 & 25 & 5.5 & 6.7 \\
\hline$p$ & 0 & 83 & 30460 & 94 & 22 & 4.7 & 24 & 6.3 & 7.2 \\
& 80 & 83 & 30460 & 96 & 22 & 3.7 & 24 & 6.5 & 7.9 \\
\hline
\end{tabular}



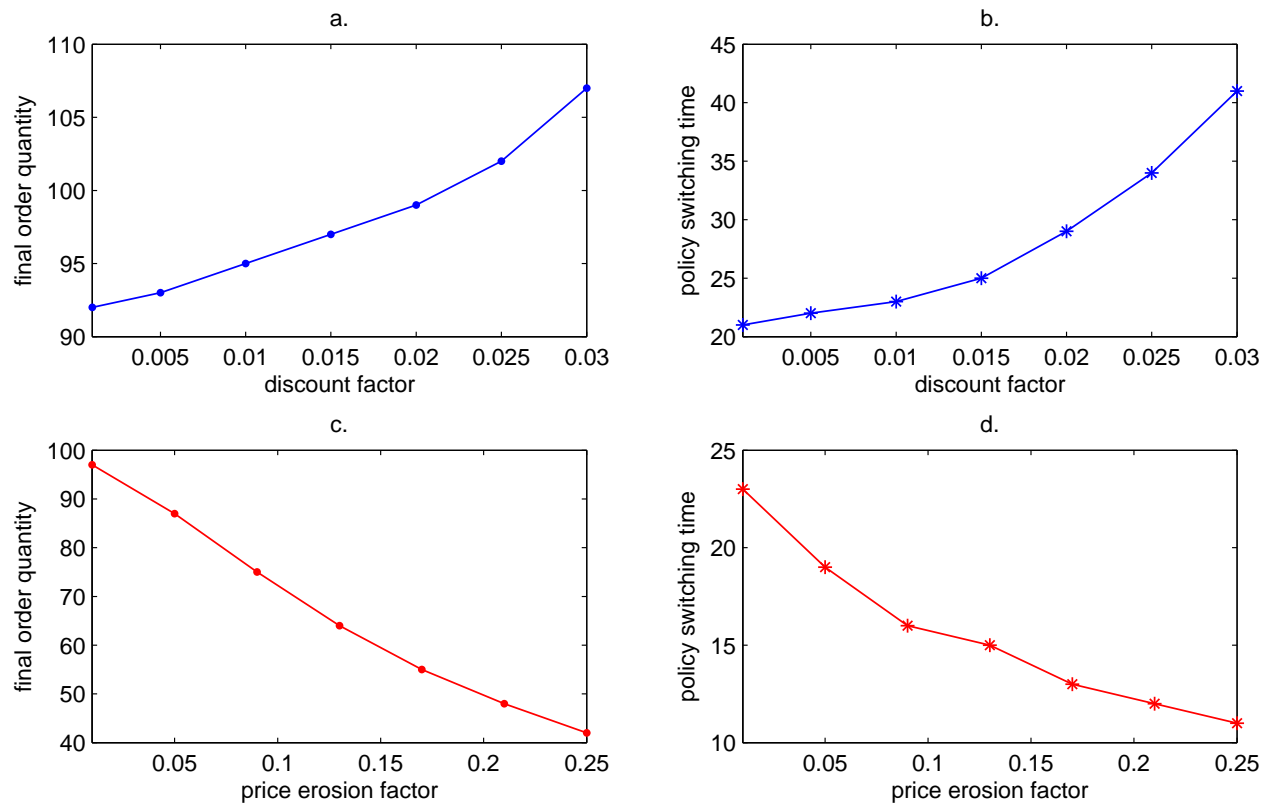

Figure 1: optimal final order quantity and time to switch versus price erosion and discount rates

consequently the final order quantity decreases (Figure 1.d). For instance, when $\gamma$ is increased to 0.25 from 0.02 , the time to switch is pushed forward to 11 from 22 and the final order quantity shrinks to 42 from 93.

It seems that the repair yield, $q$, has a high impact on the final order quantity optimal values. Basically, with the increase of the repair yield, the system resorts to ordering less in anticipation of facing more repairable parts arriving. In this case, when the system orders less the effect of purchasing cost is alleviated. Consequently, this makes the whole repair policy cheaper as opposed to the alternative policy and thus the system allows the repair policy to run for a longer period. Therefore, with the increase of the repair yield we observe an increase in the policy switching time as well.

It is also inferred from the numerical analysis that the holding and alternative policy costs are the important determinants for choosing between the repair and alternative policies. Intuitively, if holding is cheap, it is preferred to order more and switch to the alternative policy later in time. We observe when holding cost approaches zero $(h=0.25)$, then $n$ increases to 98 from 93 and $\tau$ increases to 41 from 22 in the base case. Similarly, if the alternative policy is cheap the system 


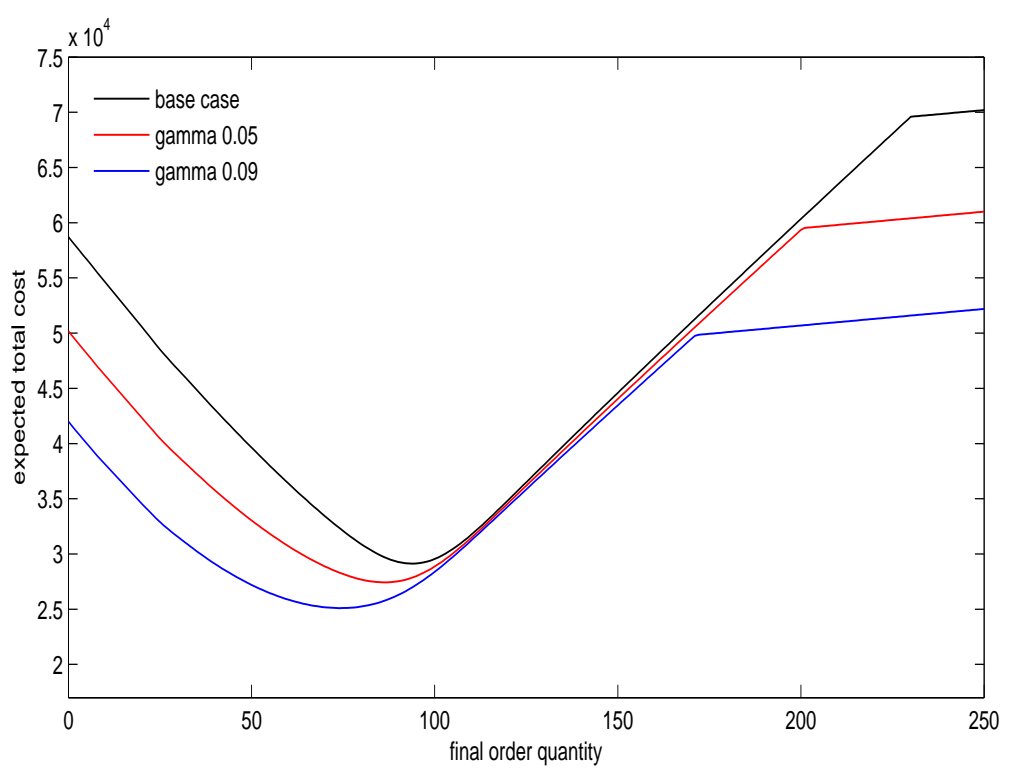

Figure 2: expected total cost versus final order quantity

orders less and the alternative policy launches earlier in order to avoid expensive repair policy associated costs.

It is observed that the repair cost does not play a major role. The intuition behind this is when repair yield is 0.1 , the repair cost just applies to a small portion of items which are repairable. Moreover, service cost does not affect the optimal value of policy switching time. The system just decides to place a slightly bigger final order quantity as it does if the service cost is cheaper. Penalty cost also has the same effect. Due to discounting, penalty cost becomes smaller over time and therefore has a minor role in determining the optimal values.

It is cumbersome to prove analytically the joint or component-wise convexity of the total cost function over $n$ and $\tau$. Figure 2 illustrates the total cost function versus the final order quantity. Apparently the expected total cost function shows a convex behavior over the final order quantity values for different discounting and price erosion rates. This is important since it justifies the use of marginal cost analysis in the first and fourth policies.

Interestingly, we also observe in figure 2 that at some point the expected total cost function starts to show a linear behavior. The reason can be explained better by also considering figure 
3. In general, when the final order quantity increases, we may expect the system to adjust the policy switching time by increasing it. But contrary to the intuition, it is not always the case and with the increase of final order quantity the system may either keep the policy switching time fixed or push it forward. This incident happens due to the fact that with the increase of the final order quantity the repair policy associated costs increase considerably. Then, in order to avoid the excessive costs that this situation imposes to the system, it becomes more cost efficient to switch to the alternative policy earlier in time and scrap the available stock on-hand. Therefore, when the final order quantity is higher than a certain value the system scraps the available serviceable inventory and retains the policy switching time at a fixed time. As a consequence we observe in the right hand side of figure 2 that at some point the expected total cost function starts to increase linearly with the scrapping cost.

From a practical standpoint, the observation in figure 3. pinpoints a drawback of applying the third policy. In this policy the system adjusts the optimal $\tau$ given the inventory level. But as it is observed, it is likely that, depending on the inventory level, the policy switching time be pushed forward or postponed and thus causes difficulties in planning for the start of the alternative policy. It is worth noting that the fourth policy does not suffer from such hassle since policy switching time is kept constant.

Another intriguing observation is that the policy without scraping (first policy) always orders less than the one with scrapping (second policy). This happens since according to the first policy the system switches to the alternative policy in case that the serviceable inventory is empty. Hence, in order to be able to switch to the alternative policy once it becomes sufficiently cheap, the system decides to order less than the second policy.

To glean an insight on the significance of the cost terms and the effect they have on the total expected cost, final order quantity and policy switching time we use a linear regression model. As listed in table 4., we deal with 10 factors affecting decision variables and objective function. To run the experiment, we assign uniformly distributed value to each factor chosen between upper and lower bounds. Then, we obtain the optimal arguments of a $(n, \tau)$ policy as well as the expected 


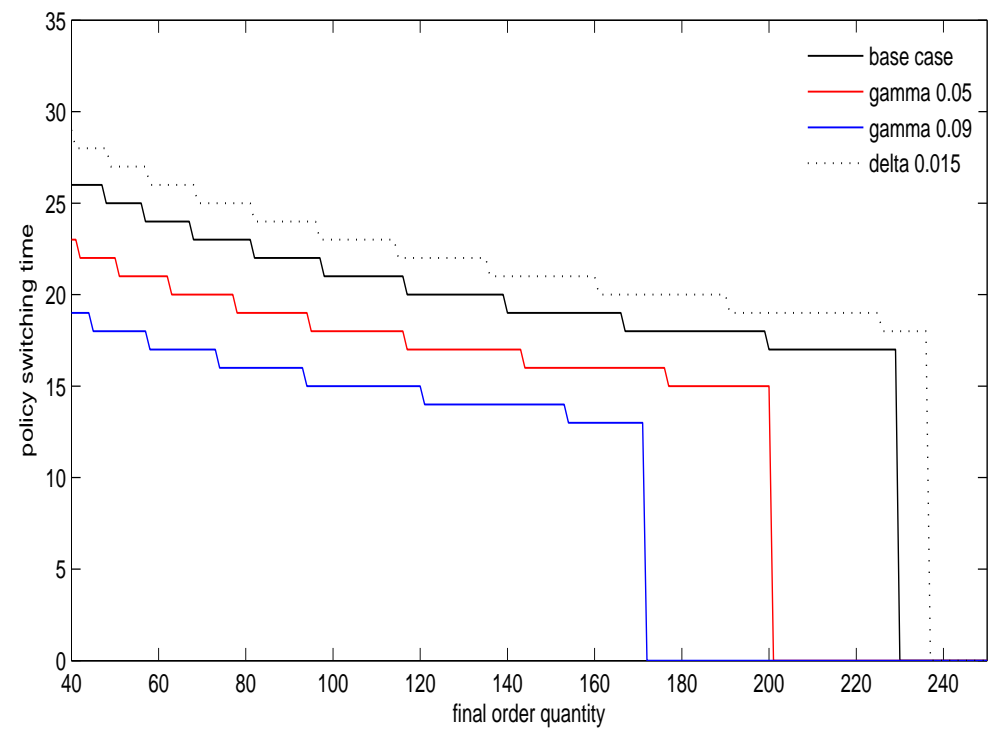

Figure 3: time to switch versus final order quantity

Table 4: Parameter values for the regression analysis

\begin{tabular}{|c|c|c|c|c|c|c|c|c|c|c|}
\hline Parameter & $\gamma$ & $\delta$ & $q$ & $c_{p}$ & $c_{a}$ & $c_{s}$ & $h$ & $c_{r}$ & $c_{s c r}$ & $p$ \\
\hline Lower bound & 0.018 & 0.005 & 0.1 & 100 & 600 & 10 & 1 & 15 & 12 & 0 \\
\hline Upper bound & 0.026 & 0.015 & 0.3 & 400 & 1000 & 50 & 4 & 35 & 55 & 30 \\
\hline
\end{tabular}

total cost corresponding to each combination of factors. Having 10 factors for which we consider two levels results in 1024 possible combinations that we use as the data set for regression analysis. Parameters and their corresponding bounds are shown in Table 4.

Price erosion rate is typically between $25 \%$ and $35 \%$ per year for the consumer electronic goods. This can be interpreted to a rate of $1.88 \%$ to $2.53 \%$ per month. The upper and lower bounds for the discount factor are set as $0.005 \%$ and $0.015 \%$. In practice about $10 \%$ of all returned spare parts are repairable. But, this value is quite dependent on the region, e.g. South America has a far higher percentage. Therefore, in the experiment repair yield factor varies between $10 \%$ and $30 \%$. It is agreed that the proposed policies, discussed in this paper, are to be used for the relatively expensive spare parts. Thus the provisioning cost chooses values between 100 and 400 . The cost of the alternative service is always more expensive than the provisioning cost, otherwise it is always 
cheaper to run the alternative policy. Hence, the bounds for the alternative cost are chosen to be 600 and 1000. Regarding the holding cost, we consider the fact that the more expensive the part is, it results in the larger holding cost components including insurance and shrinkage costs. Therefore, the magnitude of the holding cost is highly related to the provisioning cost. It is assumed that the holding cost varies between 1 to 4 per item per month.

The analysis is carried out in a 0.05 significance level $(\alpha=0.05)$. We perform three linear regressions to see the effect of cost terms on $n, \tau$ and the expected total cost. The results are listed in the appendix. Interestingly, the linear models fit the data very well with high $R^{2}$. It is observed that $c_{s c r}$ and $p$ are insignificant in determination of the total expected cost. Moreover, $\gamma, \delta, h, c_{p}$ and $q$ seem to have the highest impact on the total expected cost value. The reason that $c_{a}$ is not such an important determinant stems from the demand behavior. Due to positive skewness of the intensity function, main part of the demand happens in the earlier stages of the final phase and therefore, a minor part of the total demand is satisfied through the alternative policy. This makes $c_{a}$ to have a less significant effect on the total cost. Additionally, the parameters $c_{r}$ and $c_{s}$ seem to be insignificant in determination of $\tau$ and $n$. Furthermore, $q, c_{p}, c_{a}$ and $h$ play a significant role in determination of $\tau$ and $n$, that supports the previously mentioned observations.

\subsection{Effect of Demand Behavior}

As explained earlier we employ a non-stationary mean value function to explain demand behavior during the course of the final phase. The proposed models in the literature mostly consider a stationary demand pattern with a constant mean over time. Therefore, it might be interesting to investigate the gain that could be achieved by including an accurate non-stationary demand pattern. Denoted by intensity function 1 in figure 4., it shows the strictly decreasing poly-exponential intensity function used for the base case scenario and the corresponding mean arrival rate. However, as mentioned earlier due to the spurt in technology and innovation parts may enter the final phase while the demand rate is increasing. Therefore, we also look at an increasing and then decreasing pattern for the intensity function denoted by intensity function 2 in figure 4. 


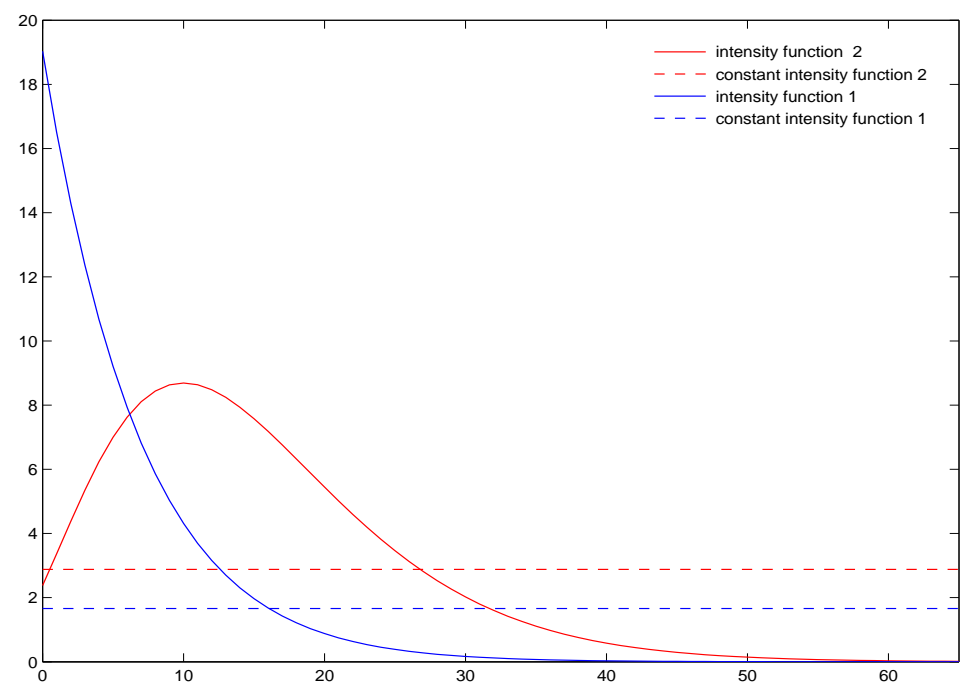

Figure 4: intensity functions

The objective is to figure out to what extend assuming a stationary demand intensity function rather than a non-stationary one affects the expected total cost of the system. To this end, we execute the second policy according to a stationary Poisson process with a constant intensity function similar to the mean intensity rate 1(2) and find the optimal values for decision variables and associated expected total cost denoted by $T C_{s}$. Then, the obtained optimal values are plugged into a model with a non-stationary arrival rate with intensity function 1(2) and the corresponding expected total cost is calculated, $T C_{n s}$. Results for different set of parameters are shown in table 5. $\Delta \%$ shows the percentage of the relative cost efficiency and is calculated according to $\Delta \%=\frac{T C_{s}-T C_{n s}}{T C_{n s}} \times 100 . \quad$ As it is observed in table 5, assuming a stationary demand pattern whereas the actual demand follows a non-stationary process considerably devastates the cost efficiency. This major observation emphasizes the prominent role that an accurate demand forecasting plays when dealing with the end-of-life inventory problem. Due to positive skewness of the intensity function, the system expects to face a big portion of the demand earlier in the course of the final phase, when repair policy is still cheaper than the alternative policy. As a consequence, always a larger final order quantity is placed in case of non-stationary demand arrival. 
Table 5: Effect of demand

\begin{tabular}{|c|c|c|c|c|c|c|c|c|c|}
\hline \multicolumn{9}{c|}{} & \multicolumn{3}{c|}{ constant intensity function 1 } & \multicolumn{2}{c|}{ intensity function 2 } & \multicolumn{2}{|c|}{ constant intensity function 2 } \\
\hline Parameter & Value & $n$ & $\tau$ & $\Delta \%$ & $n$ & $\tau$ & $n$ & $\tau$ & $(\Delta) \%$ \\
\hline$\delta$ & 0.005 & 50 & 30 & 27.13 & 131 & 33 & 72 & 25 & 15.78 \\
& 0.010 & 52 & 31 & 26.20 & 134 & 36 & 75 & 26 & 17.91 \\
& 0.015 & 54 & 33 & 25.23 & 139 & 37 & 78 & 27 & 14.85 \\
\hline$\gamma$ & 0.01 & 80 & 50 & 7.49 & 138 & 36 & 115 & 43 & 6.43 \\
& 0.05 & 24 & 13 & 28.07 & 90 & 30 & 35 & 13 & 16.68 \\
& 0.09 & 15 & 7 & 30.09 & 52 & 27 & 21 & 5 & 25.47 \\
\hline$q$ & 0.2 & 46 & 34 & 25.74 & 117 & 36 & 66 & 28 & 18.25 \\
& 0.4 & 37 & 35 & 22.31 & 88 & 40 & 54 & 29 & 19.39 \\
& 0.8 & 15 & 37 & 9.06 & 30 & 54 & 21 & 31 & 6.85 \\
\hline$c_{p}$ & 100 & 73 & 47 & 19.30 & 140 & 38 & 105 & 39 & 15.11 \\
& 350 & 32 & 23 & 15.35 & 111 & 31 & 50 & 17 & 8.12 \\
& 500 & 14 & 9 & 6.60 & 48 & 12 & 26 & 8 & 5.58 \\
\hline$c_{a}$ & 345 & 18 & 9 & 12.12 & 71 & 34 & 28 & 10 & 9.40 \\
& 945 & 71 & 45 & 24.37 & 139 & 36 & 103 & 39 & 12.42 \\
& 1245 & 81 & 57 & 20.72 & 143 & 38 & 121 & 46 & 8.69 \\
\hline$h$ & 0.24 & 71 & 46 & 16.22 & 138 & 57 & 138 & 53 & 8.71 \\
& 3.25 & 40 & 26 & 29.75 & 123 & 30 & 123 & 48 & 18.16 \\
& 6.25 & 33 & 20 & 30.69 & 114 & 27 & 114 & 44 & 16.53 \\
\hline
\end{tabular}

\section{Conclusion}

In this paper we build models to obtain the optimal final order quantity and time to switch to an alternative policy for a consumer electronics service parts in the final phase of its life cycle. The final phase starts when the part production is terminated. However, a company is mandated to serve customers due to warranty or service contract obligations. The idea of accommodating an alternative policy is triggered by the fact that consumer electronics prices erode considerably over time. Therefore, from some point in time it might be beneficial to serve demands for service parts through an alternative channel such as swapping the defective product with a new one.

To deal with this problem, we first find a closed-form expression for the expected total discounted cost in terms of elementary functions using a martingale stochastic process and related optional sampling theorem property. We then propose four policies to deal with end-of-life inventory decision making and implement them using real world data from a major European consumer electronics manufacturer. First of all, numerical analysis shows including an alternative policy for the repair is feasible and results in cost efficiency. Furthermore, it sheds light over the importance 
of various cost terms. Moreover, it shows that the "final order quantity with review and partial scrapping" outperforms all other policies in terms of cost efficiency. This is because the system avoids a build-up of excessive stocks and the associated costs by scrapping down the inventory level at each period. Moreover, the "final order quantity without review or scrapping" shows to have the worst performance.

One of the advantages of our approaches is that they inherently reduce the risk of obsolescence. All previous approaches place the final order to cover the demand over the whole service period while in our proposed approaches demand is partially serviced through part repair and therefore it considerably lowers the part obsolescence risk.

Another prominent finding lies in the importance of an accurate demand forecasting scheme in the final phase. Comparisons highlight the danger of the the assumption of a stationary demand process while demand is following a non-stationary process. Therefore, it is quite important to study the demand behavior before deciding over the final order quantity.

\section{References}

[1] Atasu, A., S. Cetinkaya. 2006. Lot Sizing for Optimal Collection and Useof Remanufacturable Returns over a Finite Life-Cycle, Production and Operations Management, 15(4), 473-487.

[2] Bradley, J.R., H.H. Guerrero. 2008. Product Design for Life Cycle Mismatch, Production and Operations Management, 17(5), Sept.-Oct. 497-512.

[3] Bradley, J.R. , H.H. Guerrero. Jan.-Feb. 2009. Life-Time Buy Decisions with Multiple Parts, Production and Operations Management, 18(1).

[4] Bulkeley, W.M. . IBM. 1999. Had '98 PC pretax loss of nearly \$1 billion, The Wall Street Journal, March 25. 
[5] Cattani, K.D. , G.C. Souza. 2003. Good buy? Delaying end-of-life purchases, European Journal of Operational Research, 146 216-228.

[6] Cinlar, E. 1975. Introduction to Stochastic Processes, Prentice-Hall, Englewood Cliffs, New Jersey.

[7] Souza, G.C. , M.E. Ketzenberg, V.D.R Guide. 2002 Capacitated Remanufacturing with SErvice Level Constraints, Production and Operations Management, 11(2) , 231-248.

[8] Fortuin, L. 1980. The All-Time Requirement of Spare Parts for Service After SalesTheoretical Analysis and Practical Results, International Journal of Operations and Production Management, 1(1), 59-70.

[9] Fortuin, L. 1981. Reduction of All-time requirements for Spare Parts, International Journal of Operations and Production Management, 2(1), 29-37.

[10] Hong, J.S. , H.Y. Koo, C.S. Lee, J. Ahn. 2008. Forecasting service parts demand for a discontinued product, IIE Transactions 40 640-649.

[11] Klein Haneveld, W.K., R.H. Teunter. 1998. The Final Order Problem, European Journal of Operational Research, 107 35-44.

[12] Liptser, R.S. , A.N. Shiryayev. 1978. Statistics of random processes. Springer Verlag, Berlin, Germany.

[13] Moore, J.R. 1971. Forecasting and Scheduling for Past-Model Replacement Parts, Management Science 18 B200-B213.

[14] Protter, P. 1992. Stochastic Integration and Differential Equations, Springer Verlag, Berlin, Germany.

[15] Ritchie, E., P. Wilcox. 1977. Renewal Theory Forecasting for Stock Control, European Jornal of operational Research Society 190-93. 
[16] Ross, S.M. 1970. Applied Probability Models with Optimization Applications, Holden-Day, San Francisco, USA.

[17] Sigar, T. 2007. Inventory Control of Spare Parts in the Final Phase, Master thesis, Erasmus University Rotterdam.

[18] Solomon, R. , P. Sandborn, M. Pecht. 2000. Electronic part life cycle concepts and obsolescence forecasting, IEEE Transactions on Components and Packaging Technologies 23 (3) 707-717.

[19] Teunter, R.H. , L. Fortuin. 1998. End-of-life service: A case study, European Journal of Operational Research, 107 19-34.

[20] Teunter, R.H. , L. Fortuin. 1999. End-of-Life Service, International Journal of Production Economics, 59 487-497.

[21] Teunter, R.H. , W.K. Klein Haneveld. 2002. Inventory control of service parts in the final phase, European Journal of Operational Research 137 497-511.

[22] Teunter, R.H., E. van der Laan and K. Inderfurth. 2000. How to set the holding cost rates in average cost inventory models with reverse logistics?. OMEGA The International Journal of Management Science 28409415.

[23] van Kooten, J.P.J., T. Tan. 2008. The Final Order Problem for Repairable Spare Parts under Condemnation. Journal of Operational Research Society.

[24] Widder, D.V. 1946. The Laplace Transform, Princeton university press, NJ, USA, 1946.

\section{A Appendix}

\section{Proof of Lemma 1.}


Proof. Since $\mathbf{N}\left(\Lambda_{1}(0)\right)=0$ and each sample path of the increasing process $t \rightarrow \mathbf{N}_{\Lambda_{1}}(t)$ is of bounded variation we obtain by Theorem $15 a$ of Widder (1972) that for every $s>0$

$$
\begin{aligned}
\delta \int_{0}^{s} \exp (-\delta t) \mathbf{N}_{\Lambda_{1}}(t) d t & =\delta \int_{0}^{s} \exp (-\delta t) \int_{0}^{t} d \mathbf{N}_{\Lambda_{1}}(v) d t \\
& =\delta \int_{0}^{s} \int_{v}^{s} \exp (-\delta t) d t d \mathbf{N}_{\Lambda_{1}}(v) \\
& =\int_{0}^{s}(\exp (-\delta v)-\exp (-\delta s)) d \mathbf{N}_{\Lambda_{1}}(v) \\
& =\int_{0}^{s} \exp (-\delta v) d \mathbf{N}_{\Lambda_{1}}(v)-\exp (-\delta s) \mathbf{N}_{\Lambda_{1}}(s)
\end{aligned}
$$

This implies

$$
\delta \int_{0}^{\tau \wedge \sigma_{n}} \exp (-\delta t) \mathbf{N}_{\Lambda_{1}}(t) d t=\int_{0}^{\tau \wedge \sigma_{n}} \exp (-\delta v) d \mathbf{N}_{\Lambda_{1}}(v)-\exp \left(-\delta\left(\tau \wedge \sigma_{n}\right)\right) \mathbf{N}_{\Lambda_{1}}\left(\tau \wedge \sigma_{n}\right)
$$

and by taking expectations the desired result follows.

\section{Proof of Lemma 2.}

Proof. By the definition of a martingale we need to verify that $\mathbb{E}\left(\mathbf{M}_{t} \mid \mathcal{F}_{s}\right) \stackrel{\text { a.s }}{=} \mathbf{M}_{s}$ for every $t \geq s$. Observe for every $t \geq s$ that

$$
\begin{aligned}
\mathbb{E}\left(\int_{0}^{t} \exp (-\alpha v) d \mathbf{N}_{\Phi}(v) \mid \mathcal{F}_{s}\right) & =\mathbb{E}\left(\int_{0}^{s} \exp (-\alpha v) d \mathbf{N}_{\Phi}(v) \mid \mathcal{F}_{s}\right)+\mathbb{E}\left(\int_{s}^{t} \exp (-\alpha v) d \mathbf{N}_{\Phi}(v) \mid \mathcal{F}_{s}\right) \\
& =\int_{0}^{s} \exp (-\alpha v) d \mathbf{N}_{\Phi}(v)+\mathbb{E}\left(\int_{s}^{t} \exp (-\alpha v) d \mathbf{N}_{\Phi}(v) \mid \mathcal{F}_{s}\right) .
\end{aligned}
$$

Since the process $\mathbf{N}_{\Phi}$ has independent increments it follows

$$
\mathbb{E}\left(\int_{s}^{t} \exp (-\alpha v) d \mathbf{N}_{\Phi}(v) \mid \mathcal{F}_{s}\right)=\mathbb{E}\left(\int_{s}^{t} \exp (-\alpha v) d \mathbf{N}_{\Phi}(v)\right)=\int_{s}^{t} \exp (-\alpha v) d \Phi(v)
$$

and this shows the desired result.

\section{Proof of Lemma 3.}


Proof. Since the random variable $\tau \wedge \sigma_{n}$ is a $\mathcal{F}_{t}$-stopping time we obtain by Lemma 2 applied to the non-stationary Poisson arrival process of non-repairable parts with mean value function $\Lambda_{1}=(1-q) \Lambda$ and the optional sampling theorem (Lipster and Shiryayev (1978)) that $\mathbb{E} \mathbf{M}_{\tau \wedge \sigma_{n}}=$ $\mathbb{E} \mathbf{M}_{0}=0$. This shows in combination with relation (4) that

$$
\begin{aligned}
\mathbb{E}\left(\int_{0}^{\tau \wedge \sigma_{n}} \exp (-\alpha t) d \mathbf{N}_{\Lambda_{1}}(t)\right) & =\mathbb{E}\left(\int_{0}^{\tau \wedge \sigma_{n}} \exp (-\alpha t) d \Lambda_{1}(t)\right) \\
& =(1-q) \mathbb{E}\left(\int_{0}^{\tau \wedge \sigma_{n}} \exp (-\alpha t) d \Lambda(t)\right) \\
& =(1-q) \mathbb{E}\left(\int_{0}^{\Lambda^{-1}\left(\Lambda(\tau) \wedge(1-q)^{-1} \mathbf{T}_{n}\right)} \exp (-\alpha t) d \Lambda(t)\right)
\end{aligned}
$$

Applying now the same argument as above to the martingale driven by the non-stationary Poisson process with mean value function $\Lambda$ yields the first formula. By a change of variables and using $\Lambda$ is strictly increasing and continuous (see Theorem 11a of Widder 1972) we also obtain

$$
\mathbb{E}\left(\int_{0}^{\Lambda^{-1}\left(\Lambda(\tau) \wedge(1-q)^{-1} \mathbf{T}_{n}\right)} \exp (-\alpha t) d \Lambda(t)\right)=\mathbb{E}\left(\int_{0}^{\Lambda(\tau) \wedge(1-q)^{-1} \mathbf{T}_{n}} \exp \left(-\alpha \Lambda^{-1}(t)\right) d t\right) .
$$

This finally shows

$$
\mathbb{E}\left(\int_{0}^{\tau \wedge \sigma_{n}} \exp (-\alpha t) d \mathbf{N}_{\Lambda}(t)\right)=\mathbb{E}\left(\int_{0}^{\Lambda(\tau) \wedge(1-q)^{-1} \mathbf{T}_{n}} \exp \left(-\alpha \Lambda^{-1}(t)\right) d t\right)
$$

and we have verified the result.

Finally the proof of Lemma 4 is given. Remark:In original proof there was a mistake in the end formula.

\section{Proof of Lemma 4.}

Proof. Clearly it follows

$$
\mathbb{E}\left(\exp \left(-\alpha\left(\tau \wedge \sigma_{n}\right)\right) \mathbf{N}_{\Lambda_{1}}\left(\tau \wedge \sigma_{n}\right)\right)=\sum_{k=0}^{n} \mathbb{E}\left(\exp \left(-\alpha\left(\tau \wedge \sigma_{n}\right)\right) \mathbf{N}_{\Lambda_{1}}\left(\tau \wedge \sigma_{n}\right) \mathbf{1}_{\left\{\mathbf{N}_{\Lambda_{1}}\left(\tau \wedge \sigma_{n}\right)=k\right\}}\right)
$$


with $\mathbf{1}_{A}$ denoting the indicator random variable of the event $A$. Since $\sigma_{n}$ denotes the random time that the process $\mathbf{N}_{\Lambda_{1}}$ hits level $n$ we obtain for every $0 \leq k \leq n-1$ that

$$
\left\{\mathbf{N}_{\Lambda_{1}}\left(\left(\tau \wedge \sigma_{n}\right)\right)=k\right\} \subseteq\left\{\sigma_{n}>\tau\right\}
$$

This shows for every $0 \leq k \leq n-1$ that

$$
\begin{aligned}
\mathbb{E}\left(\exp \left(-\delta\left(\tau \wedge \sigma_{n}\right)\right) \mathbf{N}_{\Lambda_{1}}\left(\tau \wedge \sigma_{n}\right) \mathbf{1}_{\left\{\mathbf{N}_{\Lambda_{1}}\left(\tau \wedge \sigma_{n}\right)=k\right\}}\right) & =k \mathbb{E}\left(\exp \left(-\delta\left(\tau \wedge \sigma_{n}\right)\right) \mathbf{1}_{\left\{\mathbf{N}_{\Lambda_{1}}\left(\tau \wedge \sigma_{n}\right)=k\right\}}\right) \\
& =k \exp (-\delta \tau) \mathbb{P}\left\{\mathbf{N}_{\Lambda_{1}}\left(\tau \wedge \sigma_{n}\right)=k\right\} \\
& =k \exp (-\delta \tau) \mathbb{P}\left\{\mathbf{N}_{\Lambda_{1}}(\tau)=k\right\} .
\end{aligned}
$$

Also, using $\left\{\mathbf{N}_{\Lambda_{1}}\left(\tau \wedge \sigma_{n}\right)=n\right\}=\left\{\sigma_{n} \leq \tau\right\}$, we obtain

$$
\begin{aligned}
\mathbb{E}\left(\exp \left(-\delta\left(\tau \wedge \sigma_{n}\right)\right) \mathbf{N}_{\Lambda_{1}}\left(\tau \wedge \sigma_{n}\right) \mathbf{1}_{\left\{\mathbf{N}_{\Lambda_{1}}\left(\tau \wedge \sigma_{n}\right)=n\right\}}\right) & =n \mathbb{E}\left(\exp \left(-\delta\left(\tau \wedge \sigma_{n}\right)\right) \mathbf{1}_{\left\{\sigma_{n} \leq \tau\right\}}\right) \\
& =n \mathbb{E}\left(\exp \left(-\delta \sigma_{n}\right) \mathbf{1}_{\left\{\sigma_{n} \leq \tau\right\}}\right)
\end{aligned}
$$

Combining relations (39),(40) and 41) we obtain

$$
\begin{aligned}
\mathbb{E}\left(\exp \left(-\delta\left(\tau \wedge \sigma_{n}\right)\right) \mathbf{N}_{\Lambda_{1}}\left(\tau \wedge \sigma_{n}\right)\right. & =\exp (-\delta \tau) \sum_{k=0}^{n-1} k \mathbb{P}\left\{\mathbf{N}_{\Lambda_{1}}(\tau)=k\right\}+n \mathbb{E}\left(\exp \left(-\delta \sigma_{n}\right) \mathbf{1}_{\left\{\sigma_{n} \leq \tau\right\}}\right) \\
& =\exp (-\delta \tau) \mathbb{E}\left(\mathbf{N}_{\Lambda_{1}}(\tau) \mathbf{1}_{\left\{\mathbf{N}_{\Lambda_{1}}(\tau) \leq n-1\right\}}\right)+n \mathbb{E}\left(\exp \left(-\delta \sigma_{n}\right) \mathbf{1}_{\left\{\sigma_{n} \leq \tau\right\}}\right)
\end{aligned}
$$

This shows the desired result. 
Table 6: Model summary-Expected total cost as the dependent variable

\begin{tabular}{|c|c|c|c|}
\hline R & R Square & Adjusted R Square & Std. Error of the Estimate \\
\hline 0.998 & 0.995 & 0.995 & 499.423 \\
\hline
\end{tabular}

Table 7: Coefficients-Expected total cost as the dependent variable

\begin{tabular}{|c|c|c|c|c|c|}
\hline & \multicolumn{2}{|c|}{ Unstandardized Coefficients } & Standardized Coefficients & & \\
\hline Model & $\mathrm{B}$ & Std. Error & Beta & $\mathrm{t}$ & Sig. \\
\hline (Constant) & 8099.545 & 357.427 & & 22.61 & 0.000 \\
$\gamma$ & -31029.150 & 6672.244 & -0.11 & -4.650 & 0.000 \\
$\delta$ & -37614.797 & 5951.689 & -0.15 & -6.320 & 0.000 \\
$q$ & -28937.914 & 320.558 & -0.205 & -90.274 & 0.000 \\
$h$ & 412.963 & 19.516 & 0.47 & 21.160 & 0.000 \\
$c_{p}$ & 79.136 & 0.181 & 0.971 & 437.031 & 0.000 \\
$c_{a}$ & 3.600 & 0.139 & 0.058 & 25.983 & 0.000 \\
$c_{r}$ & 22.937 & 4.929 & 0.018 & 4.654 & 0.000 \\
$c_{s}$ & 94.234 & 7.683 & 0.047 & 12.267 & 0.000 \\
$c_{s c r}$ & 2.667 & 2.199 & 0.003 & 1.213 & 0.226 \\
$p$ & 0.611 & 1.668 & 0.001 & 0.366 & 0.714 \\
\hline
\end{tabular}

Table 8: Model summary-Final order Quantity, $n$, as the dependent variable

\begin{tabular}{|c|c|c|c|}
\hline R & R Square & Adjusted R Square & Std. Error of the Estimate \\
\hline 0.994 & 0.987 & 0.987 & 0.749 \\
\hline
\end{tabular}

Table 9: Coefficients-Final order Quantity, $n$, as the dependent variable

\begin{tabular}{|c|c|c|c|c|c|}
\hline & \multicolumn{2}{|c|}{ Unstandardized Coefficients } & Standardized Coefficients & & \\
\hline Model & $\mathrm{B}$ & Std. Error & Beta & $\mathrm{t}$ & Sig. \\
\hline (Constant) & 18.877 & 0.536 & & 203.042 & 0.000 \\
$\gamma$ & -178.184 & 10.010 & -0.066 & -17.801 & 0.000 \\
$\delta$ & 43.243 & 8.929 & 0.018 & 4.824 & 0.000 \\
$q$ & -104.118 & 0.481 & -0.789 & -216.500 & 0.000 \\
$h$ & -0.653 & 0.029 & -0.080 & -22.308 & 0.000 \\
$c_{p}$ & -0.039 & 0.000 & -0.507 & -142.278 & 0.000 \\
$c_{a}$ & 0.16 & 0.000 & 0.274 & 76.944 & 0.000 \\
$c_{r}$ & -0.011 & 0.007 & -0.009 & -1.481 & 0.139 \\
$c_{s}$ & 0.002 & 0.012 & 0.001 & 0.185 & 0.853 \\
$c_{s c r}$ & -0.007 & 0.003 & -0.009 & -2.062 & 0.039 \\
$p$ & 0.017 & 0.003 & 0.028 & 6.747 & 0.000 \\
\hline
\end{tabular}


Table 10: Model summary-Policy switching time, $\tau$, as the dependent variable

\begin{tabular}{|c|c|c|c|}
\hline R & R Square & Adjusted R Square & Std. Error of the Estimate \\
\hline 0.982 & 0.964 & 0.963 & 0.432 \\
\hline
\end{tabular}

Table 11: Coefficients-Policy switching time, $\tau$, as the dependent variable

\begin{tabular}{|c|c|c|c|c|c|}
\hline & \multicolumn{2}{|c|}{ Unstandardized Coefficients } & Standardized Coefficients & & \\
\hline Model & $\mathrm{B}$ & Std. Error & Beta & $\mathrm{t}$ & Sig. \\
\hline (Constant) & 22.863 & 0.309 & & 73.938 & 0.000 \\
$\gamma$ & -130.901 & 5.772 & -0.143 & -22.677 & 0.000 \\
$\delta$ & 262.855 & 5.149 & 0.318 & 51.050 & 0.000 \\
$q$ & 15.435 & 0.277 & 0.342 & 55.657 & 0.000 \\
$h$ & -2.302 & 0.017 & -0.823 & -136.314 & 0.000 \\
$c_{p}$ & -0.003 & 0.000 & -0.106 & 17.570 & 0.000 \\
$c_{a}$ & 0.006 & 0.000 & 0.305 & 50.779 & 0.000 \\
$c_{r}$ & -0.001 & 0.004 & -0.002 & -0.229 & 0.819 \\
$c_{s}$ & -0.004 & 0.007 & -0.006 & -0.614 & 0.540 \\
$c_{s c r}$ & 0.046 & 0.002 & 0.171 & 24.175 & 0.000 \\
$p$ & -0.003 & 0.001 & -0.015 & -2.167 & 0.030 \\
\hline
\end{tabular}

